\title{
The Polycyclic Aromatic Hydrocarbon Mass Fraction on a 10 pc Scale in the Magellanic Clouds
}

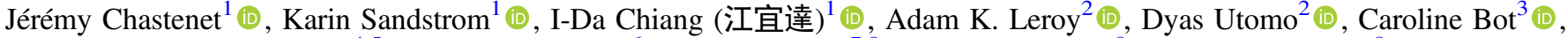 \\ Karl D. Gordon ${ }^{4,5}$ (D), Bruce T. Draine ${ }^{6}$ (D), Yasuo Fukui ${ }^{7,8}$, Toshikazu Onishi ${ }^{9}$, and Kisetsu Tsuge ${ }^{8}$ (iD \\ ${ }^{1}$ Center for Astrophysics and Space Sciences, Department of Physics, University of California, San Diego, 9500 Gilman Drive, La Jolla, CA 92093, USA \\ jchastenet@ucsd.edu \\ ${ }^{2}$ Department of Astronomy, The Ohio State University, 4055 McPherson Laboratory, 140 West 18th Ave, Columbus, OH 43210, USA \\ ${ }^{3}$ Observatoire astronomique de Strasbourg, Université de Strasbourg, CNRS, UMR 7550, 11 rue de 1' Université, F-67000 Strasbourg, France \\ ${ }^{4}$ Space Telescope Science Institute, 3700 San Martin Drive, Baltimore, MD 21218, USA \\ ${ }^{5}$ Sterrenkundig Observatorium, Universiteit Gent, Gent, Belgium \\ ${ }^{6}$ Princeton University Observatory, Peyton Hall, Princeton, NJ 08544-1001, USA \\ ${ }^{7}$ Institute for Advanced Research, Nagoya University, Furo-cho, Chikusa-ku, Nagoya 464-8601, Japan \\ ${ }^{8}$ Department of Physics, Nagoya University, Furo-cho, Chikusa-ku, Nagoya 464-8601, Japan \\ ${ }^{9}$ Department of Physical Science, Graduate School of Science, Osaka Prefecture University, 1-1 Gakuen-cho, Naka-ku, Sakai, Osaka 599-8531, Japan \\ Received 2019 February 22; revised 2019 April 3; accepted 2019 April 4; published 2019 May 6
}

\begin{abstract}
We present maps of the dust properties in the Small and Large Magellanic Clouds (SMC, LMC) from fitting Spitzer and Herschel observations with the Draine \& Li dust model. We derive the abundance of the small carbonaceous grain (or polycyclic aromatic hydrocarbon; PAH) component. The global PAH fraction $\left(q_{\mathrm{PAH}}\right.$, the fraction of the dust mass in the form of PAHs) is smaller in the SMC $\left(1.0_{-0.3}^{+0.3} \%\right)$ than in the $\mathrm{LMC}\left(3.3_{-1.3}^{+1.4} \%\right)$. We measure the PAH fraction in different gas phases ( $\mathrm{H}$ II regions, ionized gas outside of $\mathrm{H}$ II regions, molecular gas, and diffuse neutral gas). H II regions appear as distinctive holes in the spatial distribution of the PAH fraction. In both galaxies, the PAH fraction in the diffuse neutral medium is higher than in the ionized gas, but similar to the molecular gas. Even at equal radiation field intensity, the PAH fraction is lower in the ionized gas than in the diffuse neutral gas. We investigate the PAH life-cycle as a function of metallicity between the two galaxies. The PAH fraction in the diffuse neutral medium of the LMC is similar to that of the Milky Way $(\sim 4.6 \%)$, while it is significantly lower in the SMC. Plausible explanations for the higher PAH fraction in the diffuse neutral medium of the LMC compared to the SMC include: more effective PAH production by fragmentation of large grains at higher metallicity, and/or the growth of PAHs in molecular gas.
\end{abstract}

Key words: dust, extinction - ISM: abundances - Magellanic Clouds

\section{Introduction}

Dust grains have a major impact on the energy balance and chemistry of the interstellar medium (ISM), and therefore are critical to the evolution of a galaxy. Acting as a favored surface for $\mathrm{H}_{2}$ formation, they are a key agent in the chemical balance of the ISM (Le Page et al. 2009; Le Bourlot et al. 2012; Bron et al. 2014). Dust grains are also an efficient heat source for the ISM through photoelectric heating, which is the main mechanism that heats the neutral gas (Wolfire et al. 1995). The effectiveness with which dust grains play these key roles in the ISM depends on their intrinsic properties, e.g., size, charge, and chemical composition. To understand the effects of dust grains on the ISM, we need to understand their properties.

In this regard, the smallest grains are of particular interest. The small carbonaceous grain component is thought to be in the form of polycylic aromatic hydrocarbons (PAHs; Leger \& Puget 1984; Allamandola et al. 1985, 1989). PAHs play an important role in the photoelectric heating of the ISM, the efficiency of which depends on their UV absorption crosssection and grain charge (Bakes \& Tielens 1994; Weingartner \& Draine 2001b).

PAHs are also widely considered to be responsible for the mid-IR (MIR) emission features. Their emission dominates the MIR through broad emission bands at 3.3, 6.2, 7.7, 11.3, and $17 \mu \mathrm{m}$. These prominent features trace the vibrational modes of the $\mathrm{C}-\mathrm{C}, \mathrm{C}-\mathrm{C}-\mathrm{C}$, and $\mathrm{C}-\mathrm{H}$ bonds in $\mathrm{PAHs}$, and can be used to probe the ionization and size distribution of the PAH population (for a review, see Tielens 2008). Because the MIR bands from PAHs are preferentially excited by higherenergy photons, they are often considered as a tracer of star formation (Peeters et al. 2004). The intensity of some of the emission bands even allows for detection at high redshift, and can be used to determine the star formation earlier in the history of the universe (Sajina et al. 2009; Siana et al. 2009; Shipley et al. 2016). At intermediate redshifts, they are found to trace molecular gas (Cortzen et al. 2019). PAHs are also candidate carriers of the "2175 A bump," seen in extinction (Mathis 1994; Steglich et al. 2010). This intriguing feature shows variations both in width and intensity between lines of sight, between galaxies, and within the same galaxy (Gordon et al. 2003). To understand ISM thermal balance, MIR emission, and UV absorption in galaxies, we must understand the life cycle of PAHs.

Studies have found evidence of changes in PAH properties as a function of galaxy properties, particularly a PAH deficiency at low metallicity (Engelbracht et al. 2005; Madden et al. 2006; Draine et al. 2007; Galliano et al. 2008; Sandstrom et al. 2010; Paradis et al. 2011; Rémy-Ruyer et al. 2015). The intensity of the MIR features decreases in these environments relative to the total IR emission. This suggests that there is a change in the dust composition, with a lower abundance of the grains responsible for the MIR emission, compared to the larger grains emitting primarily at far-IR (FIR) wavelengths. In 
the Draine \& $\mathrm{Li}$ (2007) dust model, the PAH fraction is defined as the fraction of the total dust mass in grains with less than $10^{3}$ carbon atoms, and is hereafter labeled $q_{\mathrm{PAH}}$. The Galactic diffuse neutral medium $q_{\mathrm{PAH}}$ lies around $4.6 \%{ }^{10}$ ( $\mathrm{Li} \&$ Draine 2001; Weingartner \& Draine 2001a).

Draine et al. (2007) measured $q_{\mathrm{PAH}}$ in the SINGS galaxy sample and found a wide range of $q_{\mathrm{PAH}}$, from $\sim 0.5 \%$ in dwarf galaxies, up to almost $\sim 5 \%$ in spiral galaxies. Their results suggested a dependence between the PAH fraction and the metallicity of a galaxy, where $q_{\mathrm{PAH}}$ drops at lower metallicity. Some studies have suggested a threshold in metallicity, around $12+\log (\mathrm{O} / \mathrm{H}) \sim 8.0-8.2$, at which the $\mathrm{PAH}$ abundance varies drastically (Draine et al. 2007). On the other hand, RémyRuyer et al. (2015) find a power-law relationship between metallicity and $q_{\mathrm{PAH}}$, rather than a step function. There are several hypotheses for the dearth of PAHs in low-metallicity environments. PAHs could be exposed to more intense and/or harder far-UV radiation fields due to the overall decrease in dust shielding, and suffer from a more efficient selective photodestruction (e.g., Madden et al. 2006). PAHs could also form in the dense ISM (Zhukovska et al. 2016), and this process could be less effective at lower metallicities. Alternatively, the low PAH abundance could be the sign of a lower efficiency of forming PAH-like dust, due to particular stellar evolution at low metallicity (Galliano et al. 2008).

In order to study the metallicity trend and disentangle the various local influences on the PAH fraction in a galaxy, we focus on two of the closest galaxies, the Magellanic Clouds (MCs). The Small Magellanic Cloud (SMC) lies at about $62 \mathrm{kpc}$ (Graczyk et al. 2014), and has a metallicity of $\sim 1 / 5 Z_{\odot}$ (Russell \& Dopita 1992). The Large Magellanic Cloud (LMC) is closer, at about $50 \mathrm{kpc}$ (Walker 2012), and has a higher metallicity, of $\sim 1 / 2 Z_{\odot}$ (Russell \& Dopita 1992). The MCs therefore represent attractive targets for detailed studies of dust properties (e.g., Leroy et al. 2007; Bernard et al. 2008; Paradis et al. 2009, 2011; Bot et al. 2010; Israel et al. 2010; Sandstrom et al. 2010, 2012; Galliano et al. 2011; Gordon et al. 2014; Roman-Duval et al. 2014; Tchernyshyov et al. 2015). Their respective metallicities bracket a threshold at which $\mathrm{PAH}$ properties are thought to vary significantly (Draine et al. 2007). Here, we present a study of the PAH fraction across both MCs at $10 \mathrm{pc}$ resolution, with the same dust grain model, in order to compare to the Milky Way (MW) and other nearby galaxies. Thanks to the MCs' proximity, we can resolve ISM structures, such as H II regions, in the FIR. This allows us to provide detailed maps of $10 \mathrm{pc}$ scale PAH abundance, and investigate its variation as a function of the dominant ISM phase. Throughout this paper, we assume a constant metallicity across each galaxy. However, recent studies by Fukui et al. (2017) and Tsuge et al. (2019) suggested that the H I ridge of the LMC, south of the star-forming complex 30 Doradus, is mainly SMCstripped gas from a colliding event.

Our paper is laid out as follows: we first describe the data used in this study in Section 2. The dust grain model used to fit the dust IR emission is detailed in Section 3, and we give the results of the fit, and show the variations of the PAH fraction with environment, in Section 4. Finally, Section 5 is dedicated

\footnotetext{
${ }^{10}$ It is difficult to estimate a systematic uncertainty on $q_{\mathrm{PAH}}$. It depends on the physics of PAHs (e.g., UV, optical, and IR cross sections, and broad continuum PAH emission, which show a large scatter in their theoretical values), and modeling assumptions (e.g., starlight spectral shape).
}

to discussion and interpretation of the PAH abundance variations in the MCs.

\section{Data}

\subsection{Infrared}

We combine observations from the Spitzer Space Telescope (Werner et al. 2004) and the Herschel Space Observatory (Pilbratt et al. 2010) to cover the MIR through FIR spectral energy distribution (SED). In the MIR, we use observations from the Spitzer Legacy program SAGE (Surveying the Agents of the Galaxy), which observed both the LMC (SAGE-LMC; Meixner et al. 2006) and the SMC (SAGE-SMC; Gordon et al. 2011). The final images produced by SAGE-SMC include deeper measurements in the main star-forming regions of the SMC, from the $\mathrm{S}^{3} \mathrm{MC}$ program (Bolatto et al. 2007). In the FIR and submillimeter range, the Herschel Key Project HERITAGE (the Herschel Inventory of the Agents of Galaxy Evolution; Meixner et al. 2013, 2015) surveyed both clouds. This leads to a total of 11 photometric bands included in our study, at 3.6, 4.5, 5.8, 8.0 (Spitzer, IRAC; Fazio et al. 2004), 24, 70 (Spitzer, MIPS; Rieke et al. 2004), 100, 160 ${ }^{11}$ (Herschel, PACS; Poglitsch et al. 2010), 250, 350, and $500 \mu \mathrm{m}$ (Herschel, SPIRE; Griffin et al. 2010).

The goal of this study is to model dust emission from the MIR to the submillimeter range. However, there is a significant contribution from stars in the Spitzer IRAC bands. For the average stellar populations in a pixel, we can account for stellar emission with a simple assumption of a $5000 \mathrm{~K}$ blackbody (Draine et al. 2007, and see Section 3), but due to the proximity of the MCs, occasionally a resolution element is dominated by a very bright source that is not well modeled by a blackbody. In particular, this can be young stellar objects or evolved stars (Woods et al. 2011; Jones et al. 2017). To avoid contamination by these sources, we mask out the bright point sources that show up in the short-wavelength bands. We perform our own masking of the brightest sources in these bands in two steps. A first set of sources is simply chosen by looking at the images, and selecting the brightest stars. Second, to remove as much of the contaminating point sources as possible, we perform a fit with the unmasked images. Using these fit results, we mask stars where there is an evident bias in the parameter maps due to a point source in the image. We mask all the selected sources by replacing the value within a small radius from the source with the averaged value of the local diffuse emission.

Prior to fitting we perform several additional steps of image processing. We correct the IRAC and SPIRE images with extended source factors, as suggested by the IRAC Instrument Handbook $^{12}$ and the KINGFISH User Guide. ${ }^{13}$ Then, all observations are convolved to the SPIRE 500 resolution $\left(\sim 36^{\prime \prime}\right)$ using the Aniano et al. (2011) convolution kernels. Even though the clouds are at relatively high Galactic latitude, the observations still suffer from a non-negligible emission from the MW cirrus. We follow Gordon et al. (2014) and Chastenet et al. (2017) to remove this foreground emission. We convert the foreground H I MW column density map to a dust column density using coefficients $1.47 \times 10^{-3}, 9.32 \times 10^{-4}$,

\footnotetext{
${ }_{11}^{11}$ We use the PACS160 instead of MIPS160 band, for the sake of resolution 12 https://irsa.ipac.caltech.edu/data/SPITZER/docs/irac/ iracinstrumenthandbook/29/

${ }^{13}$ http://irsa.ipac.caltech.edu/data/Herschel/KINGFISH/docs/KINGFISH_ DR3.pdf
} 
$1.34 \times 10^{-2}, 4.28 \times 10^{-2}, 2.53 \times 10^{-2}, 2.63 \times 10^{-1}, 1.36$, $1.07,1.85,1.20,0.62$, and $0.25 \mathrm{MJy} \mathrm{sr}^{-1}\left(10^{20} \mathrm{H} \mathrm{I} \mathrm{cm}^{-2}\right)^{-1}$ from 3.6 to $500 \mu \mathrm{m}$ respectively, and subtract the foreground cirrus. We then perform a background subtraction to get rid of residual emission from the cosmic IR background, zodiacal light, and mosaicing offsets. Background regions are selected by eye: we visually identify portions of the images where we can avoid contamination from dust emission from the target galaxies. In the LMC, these are chosen to be at the edges of the images. In the SMC, we avoid the SMC bar and wing to select the background pixels. We fit and subtract a tilted plane, which removes the gradient across the background. All images are then projected with the final pixel grid sampling the pointspread function with approximately independent pixels, that is $l_{\text {pixel }} \sim 42^{\prime \prime}$, which corresponds to a pixel size of $\sim 12 \mathrm{pc}$ in the SMC and $\sim 10 \mathrm{pc}$ in the LMC. After the final projection, a background covariance matrix $\mathcal{C}_{\mathrm{bkg}}$ is constructed from the background pixels, to quantify the correlations between noise in different photometric bands (Gordon et al. 2014).

\subsection{Additional Data}

In this study, we are interested in possible correlations between the fitted dust properties and other components of the ISM. We use the SHASSA survey (the Southern H-Alpha Sky Survey Atlas; Gaustad et al. 2001) to investigate the spatial distribution of the ionized gas from $\mathrm{H} \alpha$ emission. ${ }^{14}$ We use the smoothed maps of the LMC (field 013), and SMC (field 510), at $4^{\prime}$ angular resolution. The maps are projected onto the final pixel grid of our data set, thus oversampling the point-spread function of the $\mathrm{H} \alpha$ emission data.

We use ${ }^{12} \mathrm{CO}(J=1-0)$ maps from the NANTEN (Fukui et al. 1991) survey of the SMC (Mizuno et al. 2001) and the LMC (Fukui et al. 2008) to trace the spatial distribution of the molecular gas ( $\sim 3^{\prime}$ resolution). In Section 5.2, we will use a $3 \sigma$ detection threshold in $\mathrm{CO}$ integrated intensity to define the "molecular gas phase." We determine this threshold by computing the standard deviation $\sigma$ in a region where we do not find any detections by eye. We will consider the molecular gas phase as every pixel above $3 \sigma$. In the SMC, we find a $3 \sigma$ value of $0.3 \mathrm{~K} \mathrm{~km} \mathrm{~s}^{-1}$, and $0.75 \mathrm{~K} \mathrm{~km} \mathrm{~s}^{-1}$ in the LMC. Assuming $\quad \alpha_{\mathrm{CO}}^{\mathrm{SMC}}=76 M_{\odot} \mathrm{pc}^{-2}\left(\mathrm{~K} \mathrm{~km} \mathrm{~s}^{-1}\right)^{-1}, \quad$ and $\alpha_{\mathrm{CO}}^{\mathrm{LMC}}=10 M_{\odot} \mathrm{pc}^{-2}\left(\mathrm{~K} \mathrm{~km} \mathrm{~s}^{-1}\right)^{-1}$ (Jameson et al. 2016), this corresponds to a molecular gas surface density of $22.8 M_{\odot} \mathrm{pc}^{-2}$ in the SMC, and $7.5 M_{\odot} \mathrm{pc}^{-2}$ in the LMC.

\section{SED Fitting Methodology}

We use the Draine \& $\mathrm{Li}$ (2007) model to fit the dust emission in our data set, from 3.6 to $500 \mu \mathrm{m}$ (with updates similar to those from Aniano et al. 2012). The model has a size distribution of grains divided into a carbonaceous and a silicate component. The fraction of the dust grain mass made up of PAHs with less than $10^{3}$ carbon atoms is given by the $q_{\mathrm{PAH}}$ parameter. Like Draine et al. (2007) and Aniano et al. (2012) we fit the SED using the MW $R_{\mathrm{V}}=3.1$ grain model. This model has been found to provide good fits in low-metallicity conditions by Draine et al. (2007) and Sandstrom et al. (2010) and allows us to include $q_{\mathrm{PAH}}$ as a fit parameter, which is critical to our study.

\footnotetext{
${ }^{14}$ The maps are in units of dR.1 $R=10^{6} / 4 \pi$ photons $\mathrm{cm}^{-2} \mathrm{~s}^{-1} \mathrm{sr}^{-1}$. $1 R=5.661 \times 10^{-18} \mathrm{erg} \mathrm{s}^{-1} \mathrm{~cm}^{-2} \operatorname{arcsec}^{-2}$.
}

Table 1

Fitting Parameters

\begin{tabular}{lcll}
\hline \hline Parameter & Unit & Range & Step \\
\hline$U_{\min }$ & $\ldots$ & {$[0.1,50]$} & Uneven spacing $^{\mathrm{a}}$ \\
$\log \left(\mathrm{q}_{\mathrm{PAH}}\right)$ & $\%$ & {$[-1.0,0.88]^{\mathrm{b}}$} & 0.0725 \\
$\log _{10}(\gamma)$ & $\ldots$ & {$[-3.3,0]$} & 0.1 \\
$\log _{10}\left(\Sigma_{\mathrm{d}}\right)$ & $M_{\odot} / \mathrm{pc}^{2}$ & {$[-2.0,0.7]$} & 0.15 \\
$\log _{10}\left(\Omega_{*}\right)$ & $\cdots$ & {$[-2.0,2.7]$} & 0.15 \\
\hline
\end{tabular}

Notes.

${ }^{\mathrm{a}} U_{\min } \in\{0.1,0.12,0.15,0.17,0.2,0.25,0.3,0.35,0.4,0.5,0.6,0.7,0.8,1.0$, $1.2,1.5,1.7,2.0,2.5,3.0,3.5,4.0,5.0,6.0,7.0,8.0,10.0,12.0,15.0,17.0$, $20.0,25.0,30.0,35.0,40.0,50.0\}$.

${ }^{\mathrm{b}}$ We also include $\mathrm{q}_{\mathrm{PAH}}=0$.

In each pixel $j$, the dust is heated by a range of radiation field intensities described by the parameter $U$, a dimensionless factor scaling the Mathis et al. (1983) $10 \mathrm{kpc}$ MW interstellar radiation field. In each pixel, a fraction $(1-\gamma)$ of the dust grain mass is heated by a radiation field of intensity $U_{\min }$. The remaining fraction $\gamma$ is heated by a power-law distribution of $U_{\min } \leqslant U \leqslant U_{\max }$ (see Equations (8)-(10) of Aniano et al. 2012), with a power-law exponent $\alpha$. In this study, we fix $U_{\max }=10^{7}$, and $\alpha=2$. In the end, we have five free parameters: the minimum radiation field $U_{\min }, \gamma$ the fraction of the dust mass heated by the power-law distribution of radiation fields, the PAH fraction $q_{\mathrm{PAH}}$, the dust surface density $\Sigma_{\mathrm{d}}$, and the scaling parameter of stellar surface brightness, $\Omega_{*}$, which adjusts a $5000 \mathrm{~K}$ blackbody to match the observed starlight continuum in the shortest-wavelength bands. See Table 1 for the boundaries of each parameter (Range), and the sampling (Step). The parameter values ensure a sampling fine enough to resolve the 1D likelihood functions and were determined after several iterations of the fit.

Using the fitted parameters, we calculate, in each pixel $j, \bar{U}$ and $f_{\mathrm{PDR}}$, as described by Aniano et al. (2012). $\bar{U}$ measures the dust-mass-weighted average radiation field intensity, generally given by

$$
\begin{aligned}
\overline{U_{j}}= & \left(1-\gamma_{j}\right) U_{\min , j}+\gamma_{j} \\
& \times\left\{\begin{array}{l}
\left(\frac{\alpha_{j}-1}{\alpha_{j}-2}\right)\left(\frac{U_{\max }^{2-\alpha_{j}}-U_{\min , j}^{2-\alpha_{j}}}{U_{\max }^{1-\alpha_{j}}-U_{\min , j}^{1-\alpha_{j}}}\right), \text { if } \alpha \neq 1, \alpha \neq 2 ; \\
U_{\min , j} \frac{\ln \left(U_{\max } / U_{\min j}\right)}{1-U_{\min , j} / U_{\max }}, \text { if } \alpha=2 .
\end{array}\right.
\end{aligned}
$$

$f_{\mathrm{PDR}}$ is the fraction of the dust luminosity produced by regions where $U \geqslant U_{\mathrm{PDR}}=10^{2}$ :

$$
f_{\mathrm{PDR}}=L_{\mathrm{PDR}} / L_{\mathrm{dust}}
$$

with

$$
\begin{aligned}
& L_{\mathrm{PDR}, j}=P_{0, j}\left(q_{\mathrm{PAH}}\right) M_{\mathrm{d}, j} \gamma_{j} \frac{\ln \left(U_{\mathrm{max}} / U_{\mathrm{PDR}}\right)}{U_{\min , j}^{-1}-U_{\max }^{-1}}, \\
& L_{\mathrm{dust}, j}=P_{0, j}\left(q_{\mathrm{PAH}}\right) M_{\mathrm{d}, j} \overline{U_{j}} .
\end{aligned}
$$

Here $P_{0}\left(q_{\mathrm{PAH}}\right)$ is the power radiated per unit dust mass, when $U=1$, and $M_{\mathrm{d}}$ is the dust mass.

Aside from their pixel-to-pixel distribution, we measure galaxy-averaged values of the fitted parameters, weighted 


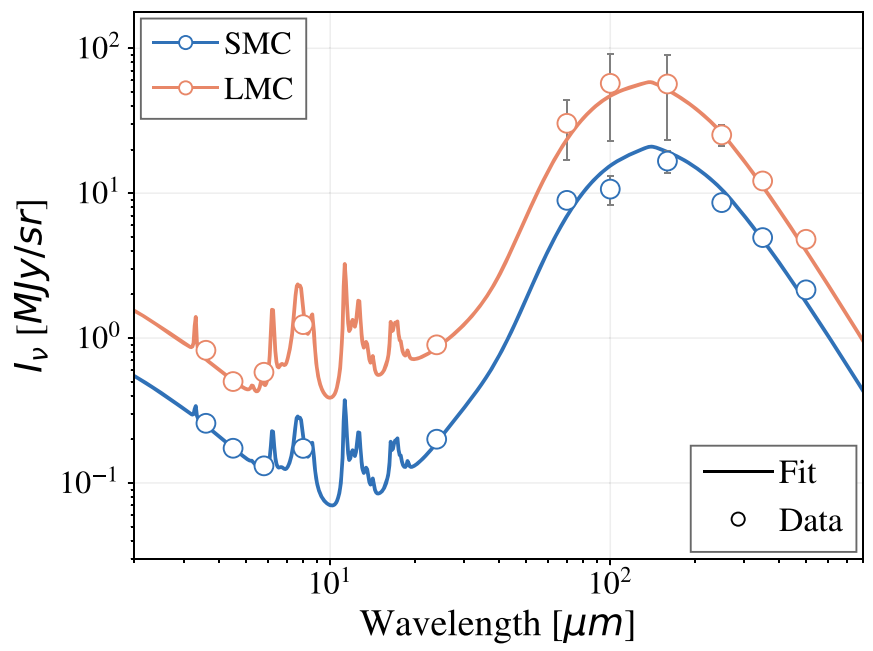

Figure 1. Examples of the fitting results in two pixels in the diffuse ISM of the SMC (blue) and the LMC (orange). The circles mark the data spectral energy distributions with their $3 \sigma$ errors, and the solid lines show the best-fit model in each case.

according to the dust mass or luminosity distribution. Following Aniano et al. (2012), we define the dust-mass averaged value of the mass fraction of PAHs,

$$
\left\langle q_{\mathrm{PAH}}\right\rangle=\frac{\sum_{j}^{N} q_{\mathrm{PAH}, j} M_{\mathrm{d}, j}}{\sum_{j}^{N} M_{\mathrm{d}, j}},
$$

and similarly, the dust-mass averaged starlight intensity,

$$
\langle\bar{U}\rangle=\frac{\sum_{j}^{N} \bar{U}_{j} M_{\mathrm{d}, j}}{\sum_{j}^{N} M_{\mathrm{d}, j}} .
$$

The average value of $f_{\mathrm{PDR}}$, i.e., dust luminosity weighted, is expressed as

$$
\left\langle f_{\mathrm{PDR}}\right\rangle=\frac{\sum_{j}^{N} L_{\mathrm{PDR}, j}}{\sum_{j}^{N} L_{\mathrm{dust}, j}} .
$$

We assume that $P_{0}\left(q_{\mathrm{PAH}}\right)$ variations are small enough pixel-topixel to be negligible (Aniano et al. 2012).

\subsection{Fitting and Uncertainties}

In Figure 1, we show two examples of the data in the SMC and the LMC fit by the best model (i.e., maximum likelihood), in two pixels of the diffuse ISM. The error bars at short wavelengths show that the errors are small enough to strongly constrain $q_{\mathrm{PAH}}$. Residuals show that the fits are good in both galaxies at short wavelengths, especially $8 \mu \mathrm{m}$. At long wavelengths, the fractional residuals are mostly negative, indicating that the model generally overestimates the data (residuals peak at less than $10 \%$ ).

The fitting is done with the DustBFF tool (Gordon et al. 2014), which determines the $n$-dimensional a posteriori likelihood distribution of the parameters. This is a Bayesian fitting tool that uses flat priors on the parameter distributions, and a covariance matrix, built from background pixels and instrument errors, to propagate uncertainties. We use the same calibration errors on Spitzer and Herschel instruments as those in Gordon et al. (2014) and Chastenet et al. (2017). The diagonal elements of the covariance matrix include both uncorrelated (or statistical) and correlated errors, and the non-diagonal elements measure only correlated errors between the photometric bands. We refer the reader to Gordon et al. (2014) for further details on the covariance matrix and the DustBFF fitting.

We use the five-dimensional (for five fitting parameters) likelihood function to build realizations of the parameter maps: a realization is a sample of the five-dimensional grid, weighted by the likelihood function. The realizations render noise properties more accurately than finding only the maximum of the likelihood function. To measure values like $\left\langle q_{\mathrm{PAH}}\right\rangle$ and their associated 16th and 84th percentiles, we prefer the realization method over the expectation value (Gordon et al. 2014; Chiang et al. 2018; Utomo et al. 2019), since it draws samples without marginalizing the likelihood over all but one parameter dimension. Since we compute weighted values with both $q_{\mathrm{PAH}}$ and $\Sigma_{\mathrm{d}}$, it is better to use the full-dimension likelihood distribution to avoid losing information. We build a large number of realization maps for each parameter simultaneously (both the fitted parameters and the calculated parameters like $\bar{U}$ and $\left.f_{\mathrm{PDR}}\right)$, and use them to calculate the mean in each pixel. These are presented in Figures 2 and 3.

From the realizations of a single pixel or group of pixels, we can determine their statistical error by measuring the standard deviation from a large number of realizations. When combining many pixels together we can propagate these uncertainties to calculate the error on the mean values. To represent the intrinsic scatter of a parameter within a specific region of the galaxy (e.g., Figures 7 or 8), we use the 16th and 84th percentiles of the dust mass- (or dust luminosity for $f_{\mathrm{PDR}}$ )-weighted distribution of said parameter. For all averaged parameters, e.g., $\left\langle q_{\mathrm{PAH}}\right\rangle$, quoted in the rest of the paper, the statistical uncertainties are generally very small, due to the large numbers of pixels begin averaged together. We instead list the \pm the 16th and 84th percentiles of the dust mass- or luminosity-weighted distributions.

\section{Results \\ 4.1. Fitting Parameter Maps}

Figures 2 and 3 show the results from fitting the pixels of the SMC and the LMC maps, respectively, as well as the computed values of $\bar{U}$ and $f_{\mathrm{PDR}}$, as described in Section 3. The overall distribution of $\Sigma_{\mathrm{d}}$ shifts toward higher values in the LMC than in the SMC, as expected because of the larger dust-to-gas ratio of the former (Gordon et al. 2014). A significant difference is also seen in the $q_{\mathrm{PAH}}$ parameter, which is clearly higher in the LMC than in the SMC (a larger version of the $q_{\mathrm{PAH}}$ map is shown in Figure 4). We find dust-mass-weighted averages for each galaxy of

$$
\begin{aligned}
& \left\langle q_{\mathrm{PAH}}^{\mathrm{SMC}}\right\rangle=1.0_{-0.3}^{+0.3} \%, \text { and } \\
& \left\langle q_{\mathrm{PAH}}^{\mathrm{LMC}}\right\rangle=3.3_{-1.3}^{+1.4} \% .
\end{aligned}
$$

Both of these values fall within the range seen by Draine et al. (2007) in the SINGS sample at the relevant metallicities. By studying a sample of low-metallicity galaxies, Rémy-Ruyer et al. (2015) found a power-law relation between the metallicity and the PAH fraction (see their Equation (5)). If we apply this relation to the MCs' metallicities (SMC: $12+\log (\mathrm{O} / \mathrm{H}) \sim 8.1$; 


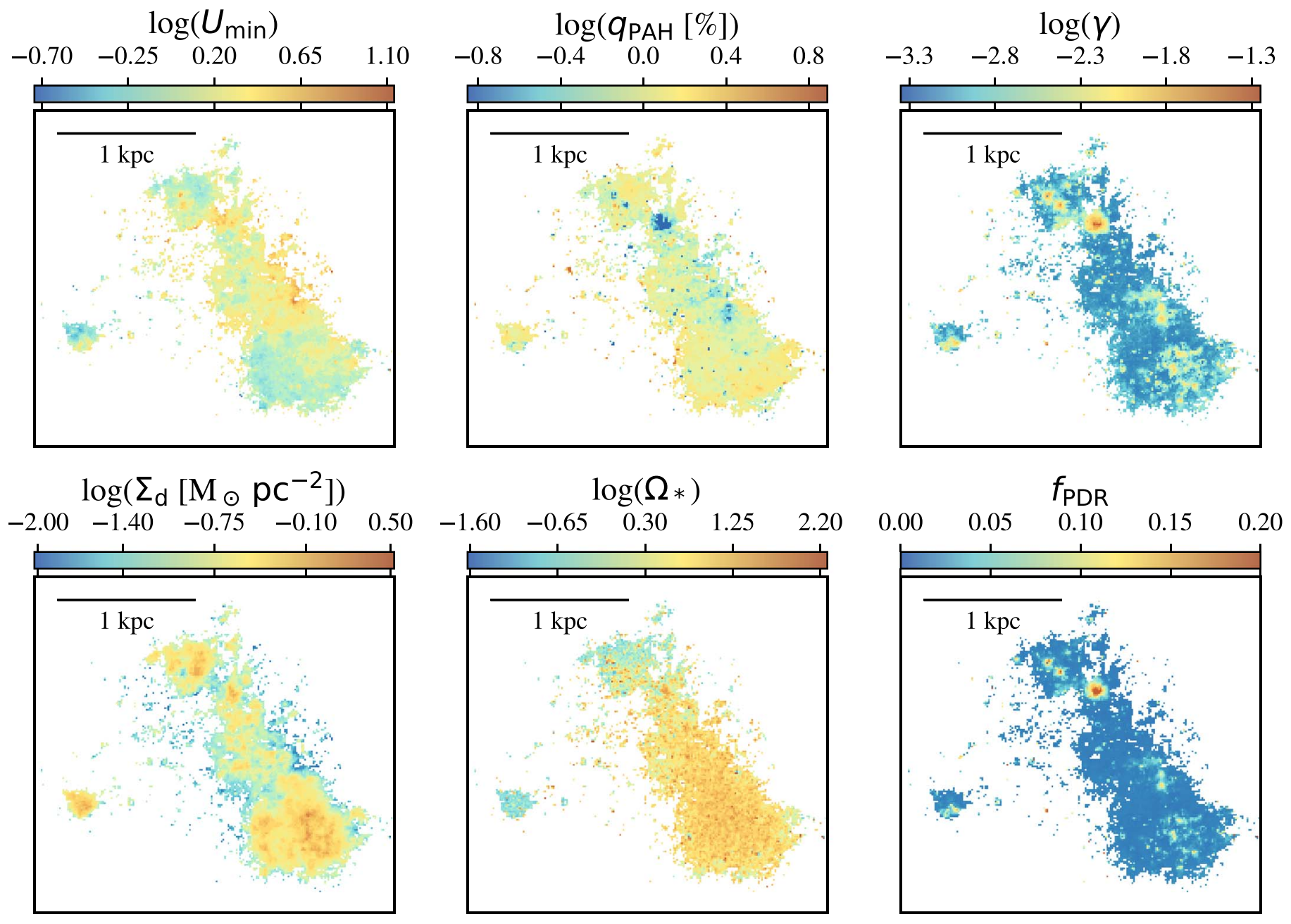

Figure 2. Results of the fitting in the SMC for the minimum radiation field, $U_{\min }$, the PAH mass fraction, $q_{\mathrm{PAH}}$ (see Figure 4 and Section 4.2 ), the weight of dust mass heated by a power-law combination of radiation field, $\gamma$, the total dust surface density, $\Sigma_{\mathrm{d}}$, the scaling of stellar surface brightness, $\Omega_{*}$, and the derived fraction of dust luminosity where $U>10^{2}, f_{\mathrm{PDR}}$.

LMC: $12+\log (\mathrm{O} / \mathrm{H}) \sim 8.3$; Russell \& Dopita 1992), we find

$$
\begin{aligned}
& 0.69 \% \leqslant q_{\mathrm{PAH}}^{\mathrm{RR} 15}(\mathrm{SMC}) \leqslant 3.47 \%, \text { and } \\
& 1.25 \% \leqslant q_{\mathrm{PAH}}^{\mathrm{RR} 15}(\mathrm{LMC}) \leqslant 6.31 \% .
\end{aligned}
$$

Our average PAH fractions found in this work fall well within these ranges as well.

In both galaxies over most of the area, $U_{\min } \sim \bar{U}$. Thus we only show the fitting parameter $U_{\min }$ in Figures 2 and 3 . We calculate the dust-mass-weighted average of $\bar{U}$ and find $\langle\bar{U}\rangle=$ $1.2_{-0.4}^{+0.4}$ in the SMC and $\langle\bar{U}\rangle=1.6_{-1.0}^{+1.6}$ in the LMC. Utomo et al. (2019) recently studied the distributions of mass and temperature in four nearby galaxies, including the SMC and the LMC, using a single-temperature modified blackbody model. They found that the distribution of dust mass as a function of radiation field intensity peaks at values of $U_{\text {peak }}=1.1$ in the SMC and $U_{\text {peak }}=1.8$ in the LMC, consistent with our results.

Sandstrom et al. (2010) found a dust-mass-weighted PAH fraction of $\sim 0.6 \%$ in the SMC (compared to the $1.0 \%$ in this study). One possible explanation for our lower fraction is the limited coverage of the dust emission SED in Sandstrom et al. (2010) compared to this paper: here, the longest wavelength is $500 \mu \mathrm{m}$ while it is $160 \mu \mathrm{m}$ in Sandstrom et al. With the addition of the Herschel bands, we are able to better constrain the total dust mass and temperature, particularly in regions with colder dust, affecting the PAH fraction. In addition, the extent of the $\mathrm{S}^{3} \mathrm{MC}$ Spitzer maps used in that paper did not allow as accurate a MW foreground removal as enabled by the expanded SAGE-SMC coverage. This may have resulted in an oversubtraction of actual SMC emission in the MIR bands, decreasing the Sandstrom et al. (2010) $q_{\mathrm{PAH}}$ value.

In the LMC, Paradis et al. (2009) found an enhanced PAH fraction with respect to the large grain abundance in the stellar bar. Our results do not show an increased $q_{\mathrm{PAH}}$ in this region, as other regions of the LMC show the same PAH fraction as in the optical bar. This difference could be the result of including the Herschel bands, and thereby more accurately measuring $q_{\mathrm{PAH}}$. It may also be the result of using a different dust model (e.g., the Desert et al. 1990 model in the paper by Paradis et al. 2009), or a different stellar continuum model.

We find values of the global PDR fraction (i.e., the fraction of the dust luminosity produced by regions where $U \geqslant 10^{2}$ ):

$$
\begin{aligned}
& \left\langle f_{\mathrm{PDR}}^{\mathrm{SMC}}\right\rangle=3.29_{-0.02}^{+0.01} \%, \text { and } \\
& \left\langle f_{\mathrm{PDR}}^{\mathrm{LMC}}\right\rangle=9.17_{-0.04}^{+0.04} \% .
\end{aligned}
$$

These values are below the average values found in NGC 628 and NGC 6946, two nearby, resolved galaxies, by Aniano et al. 


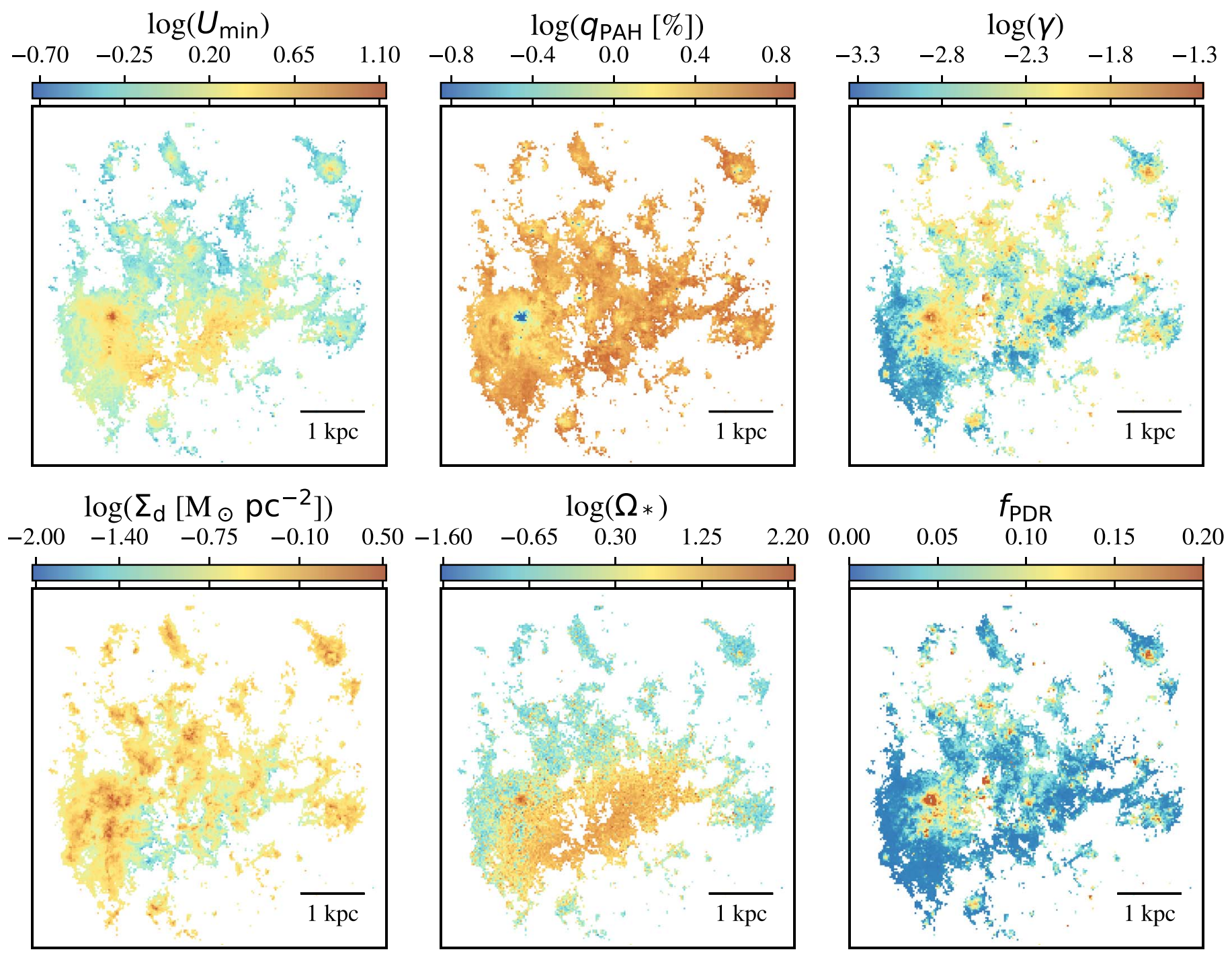

Figure 3. Results of the fitting in the LMC for $U_{\min }, q_{\mathrm{PAH}}, \gamma, \Sigma_{\mathrm{d}}$, and $\Omega_{*}$, and the derived $f_{\mathrm{PDR}}$.

(2012; 11\%). However, this is not surprising as the spatial scales in their study are coarser than for the MCs: due to limited resolution, the luminosity-weighted $f_{\mathrm{PDR}}$ is biased toward highluminosity values. The resulting weighted average is then more sensitive to these high-luminosity regions due to the blending of signal. This is one of the key results of the recent study by Utomo et al. (2019). We do find high $f_{\mathrm{PDR}}$ values in starforming regions, up to $\sim 60 \%$ in 30 Dor (LMC), and $\sim 50 \%$ in N66 (SMC).

The spatial distributions of $\Omega_{*}$ trace the regions with high stellar density (Zaritsky et al. 2002, 2004). They show the old stellar spheroid population of the SMC, as well as the optical bar in the LMC.

\subsection{PAHs in the MCs}

In Figure 4, we show the maps of the $q_{\mathrm{PAH}}$ parameter in the LMC (top) and the SMC (bottom). The contours are $\mathrm{H} \alpha$ emission from the SHASSA survey (Gaustad et al. 2001) at level of $\sim 1.5 \times 10^{-16} \mathrm{erg} \mathrm{s}^{-1} \mathrm{~cm}^{-2} \operatorname{arcsec}^{-2}(\sim 300 \mathrm{dR}$; solid line; the reason for choosing this value is discussed later in this section). The labeled circles are $\mathrm{H}$ II regions as identified in Lopez et al. (2014); the radii correspond to those given in their
Table 1, and are defined as the limit where they enclose $90 \%$ of the $\mathrm{H} \alpha$ emission of the source. There are other known H II regions in the MCs; however, identification of $\mathrm{H}$ II regions and their boundaries is not straightforward, and we use only those in Lopez et al. (2014) for the sake of homogeneity. From these maps we draw several conclusions which we discuss in the following (sub)sections: (1) $q_{\mathrm{PAH}}$ varies dramatically between the SMC and LMC, (2) $q_{\mathrm{PAH}}$ shows variations within each galaxy, and (3) a primary driver for the variation in $q_{\mathrm{PAH}}$ within each galaxy appears to be correlated with the presence of ionized gas as traced by $\mathrm{H} \alpha$.

To investigate the variations of $q_{\mathrm{PAH}}$ within and between the galaxies, we make four separations with respect to the ISM gas phase. In Table 2, we report $\left\langle q_{\mathrm{PAH}}\right\rangle$ in these four phases. There is no overlap between these values as we use only pixels that do not fall in two gas phase definitions. In short, we observe the following:

$$
q_{\mathrm{PAH}}^{\text {mol }} \sim q_{\mathrm{PAH}}^{\text {diff. neutr. }}>q_{\mathrm{PAH}}^{\text {out-H II }}>q_{\mathrm{PAH}}^{\mathrm{H} \mathrm{II}} .
$$

The implications of these observations for the PAH life-cycle will be discussed further in Section 5.2. We define the four phases as follows. 

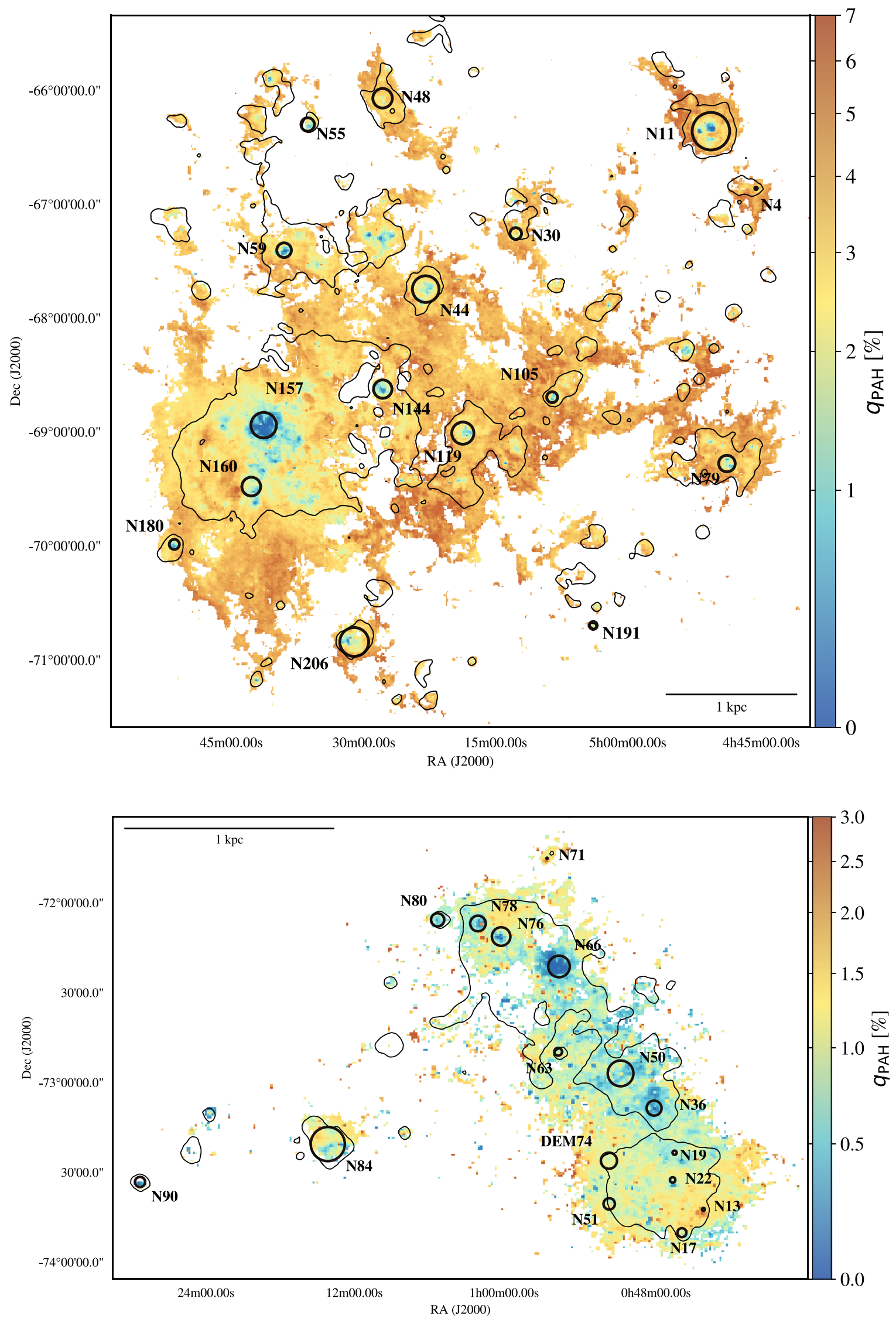

Figure 4. Maps of the $q_{\mathrm{PAH}}$ parameter in the LMC (top) and the SMC (bottom). We find dust-mass-averaged values of $\left\langle q_{\mathrm{PAH}}^{\mathrm{SMC}}\right\rangle=1.0 \%$, and $\left\langle q_{\mathrm{PAH}}^{\mathrm{LMC}}\right\rangle=3.3 \%$. The contours are $\mathrm{H} \alpha$ emission from the SHASSA survey at $\sim 1.5 \times 10^{-16} \mathrm{erg} \mathrm{s}^{-1} \mathrm{~cm}^{-2} \operatorname{arcsec}^{-2}$. The labeled circles are H II regions identified by Lopez et al. (2014). See Section 4.2 for details. 
Table 2

$\left\langle q_{\mathrm{PAH}}\right\rangle$ (in \%) in Different Gas Phases of Each Galaxy

\begin{tabular}{lcc}
\hline \hline & SMC & LMC \\
\hline Global Average & $1.0_{-0.3}^{+0.3}$ & $3.3_{-1.3}^{+1.4}$ \\
\hline H II regions & $0.8_{-0.5}^{+0.3}$ & $1.8_{-1.3}^{+1.1}$ \\
Non-H II region ionized gas $^{\mathrm{a}}$ & $0.9_{-0.3}^{+0.3}$ & $2.9_{-1.2}^{+1.1}$ \\
Diffuse neutral $^{\mathrm{b}}$ & $1.1_{-0.3}^{+0.2}$ & $4.1_{-0.8}^{+0.6}$ \\
Molecular gas $^{\mathrm{c}}$ & $1.1_{-0.2}^{+0.1}$ & $4.3_{-0.9}^{+1.3}$ \\
\hline
\end{tabular}

Notes.

a All pixels above the lower limit in $\mathrm{H} \alpha$, excluding $\mathrm{H}$ II regions.

${ }^{b}$ All pixels below the $\mathrm{CO}$ detection, the limit in $\mathrm{H} \alpha$, and outside of $\mathrm{H} \mathrm{II}$ regions.

c All pixels with $\mathrm{CO}$ detection not overlapping with pixels with $I_{\mathrm{H} \alpha} \gtrsim 1.5 \times 10^{-16} \mathrm{erg} \mathrm{s}^{-1} \mathrm{~cm}^{-2} \operatorname{arcsec}^{-2}$.

1. Ionized gas toward H II regions. This is simply defined by the pixels falling within the radii of $\mathrm{H}$ II regions from Lopez et al. (2014). In the LMC, there is a clear drop in the dust-mass-weighted PAH fraction, and $\left\langle q_{\mathrm{PAH}}^{\mathrm{H} \text { II }}\right\rangle$ reaches only $\sim 1.8_{-1.3}^{+1.1} \%$, i.e., slightly more than half of the galaxy average. In the SMC, the dust-mass-weighted PAH fraction reaches $\sim 0.8_{-0.5}^{+0.3} \%$. We note that the harder radiation field in and near $\mathrm{H}$ II regions, which is not taken into account in our fitting, would lead us to overestimate $q_{\mathrm{PAH}}$ in these regions. The values found here are therefore conservative.

2. Non-H II region ionized gas. We distinguish the ionized gas inside and outside $\mathrm{H}$ II regions, by selecting pixels whose $\mathrm{H} \alpha$ surface brightness is above $I_{\mathrm{H} \alpha} \sim 1.5 \times 10^{-16} \mathrm{erg} \mathrm{s}^{-1} \mathrm{~cm}^{-2} \operatorname{arcsec}^{-2}$ but not in identified $\mathrm{H}$ II regions. Although this ionized gas is in a more diffuse phase than the gas in H II regions, we avoid identifying it as "diffuse ionized gas (DIG)" due to the specific ways that DIG is defined in nearby galaxies (for reviews, see Mathis 2000; Haffner et al. 2009, and references therein). We discuss this further in Section 5.3. We find $\left\langle q_{\mathrm{PAH}}^{\mathrm{out}-\mathrm{H} \text { II }}\right\rangle=2.9_{-1.2}^{+1.1} \%$ in the $\mathrm{LMC}$ and $\left\langle q_{\mathrm{PAH}}^{\mathrm{out}-\mathrm{H} \mathrm{II}}\right\rangle=0.9_{-0.3}^{+0.3} \%$ in the SMC.

3. Molecular gas. We use ${ }^{12} \mathrm{CO}(J=1-0)$ maps (Section 2) to trace the molecular gas. We define this phase with every pixel above the $3 \sigma$ detection threshold, and $I_{\mathrm{H} \alpha} \lesssim 1.5 \times 10^{-16} \mathrm{erg} \mathrm{s}^{-1} \mathrm{~cm}^{-2} \operatorname{arcsec}^{-2}$. We find $\left\langle q_{\mathrm{PAH}}^{\text {mol. }}\right\rangle \sim 4.3_{-0.9}^{+1.3} \%$ in the LMC, and $\left\langle q_{\mathrm{PAH}}^{\mathrm{mol}}\right\rangle \sim 1.1_{-0.2}^{+0.1} \%$ in the SMC, similar to the values in the diffuse neutral medium.

4. Diffuse neutral gas. This is defined with the regions that fall into none of the above categories: with $\mathrm{H} \alpha$ emission lower than $\sim 1.5 \times 10^{-16} \mathrm{erg} \mathrm{s}^{-1} \mathrm{~cm}^{-2} \operatorname{arcsec}^{-2}$, which also means outside of an $\mathrm{HII}$ region, and below the $3 \sigma$ $\mathrm{CO}$ detection. We use the dust-mass-weighted PAH fraction in the diffuse neutral medium as a reference value for each cloud. We find $\left\langle q_{\mathrm{PAH}}^{\text {ref }}\right\rangle=1.1_{-0.3}^{+0.2} \%$ in the $\mathrm{SMC}$, and $\left\langle q_{\mathrm{PAH}}^{\mathrm{ref}}\right\rangle=4.1_{-0.8}^{+0.6} \%$ in the LMC.

\section{3. $q_{\mathrm{PAH}}$ in H II Regions}

The H II regions, as identified in Lopez et al. (2014), appear as minima in the $q_{\mathrm{PAH}}$ map, and suggest that PAHs are destroyed inside $\mathrm{H}$ II regions. In Figure 4, we can see that most
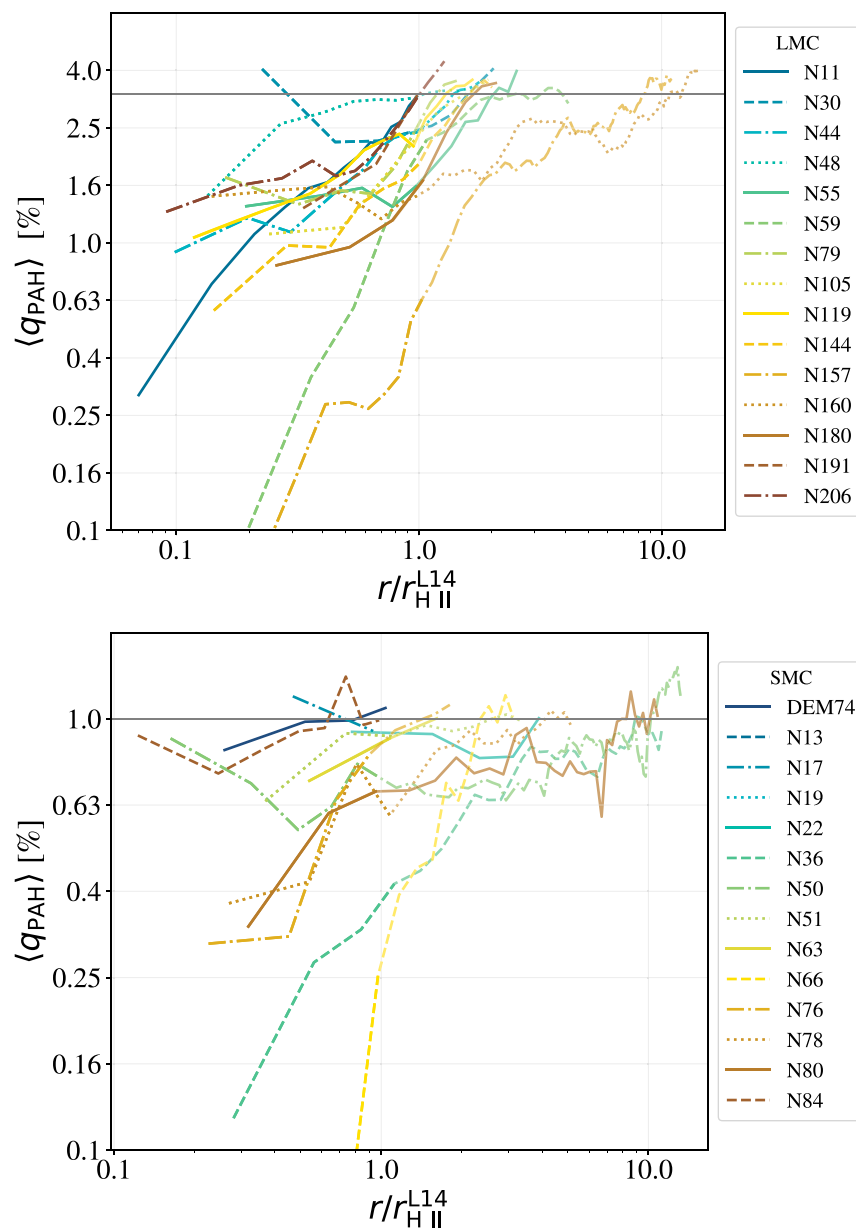

Figure 5. Radial profiles of $\left\langle q_{\mathrm{PAH}}\right\rangle$ in the LMC (top) and the SMC (bottom), from the centers of each $\mathrm{H}$ II region, normalized by the radius given by Lopez et al. (2014). The horizontal gray lines mark the average for each galaxy. The maximum radii are chosen to be slightly higher than the radii of the spheres of influence, in order to show their full extent.

H II regions, marked by the black labeled circles, are indeed low in PAHs, with respect to the abundance in the other parts of each galaxy.

We are interested in understanding how $\mathrm{H}$ II regions affect the PAH fraction. In Figure 5, we show the radial profiles of $\left\langle q_{\mathrm{PAH}}\right\rangle$ for each $\mathrm{H}$ II region in the SMC (bottom) and the LMC (top). We see that $\left\langle q_{\text {PAH }}\right\rangle$ drops to very low values only in a handful of cases (notably, $0 \%$ in 30 Dor). This is expected, as the diffuse neutral gas projected along the line of sight contaminates our measurement of the $q_{\mathrm{PAH}}$ inside the H II region. The dust emission along the line of sight reflects the full column through the ISM of the galaxy, not just the H II region, so we do not expect the observed $q_{\mathrm{PAH}}$ to be $0 \%$. In the particular case of 30 Dor, a result consistent with $0 \%$ PAH fraction could mean that the actual $\mathrm{H}$ II region dominates the entire line of sight through the LMC.

For each $\mathrm{H}$ II region, we measure the radius at which $\left\langle q_{\mathrm{PAH}}\right\rangle$ goes back to the global average (horizontal gray line in Figure 5). In this figure, we see that $\left\langle q_{\mathrm{PAH}}\right\rangle$ in each $\mathrm{H}$ II region goes back to the galaxy average at different radii. We treat these radii as the "spheres of influence" of each H II region on the surrounding PAH fraction. In Figure 6, we plot these radii against the total $\mathrm{H} \alpha$ luminosity from within that radius. We find that these radii correlate very well with $\mathrm{H} \alpha$ luminosity with a power-law coefficient between 0.35 and 0.4 in both 


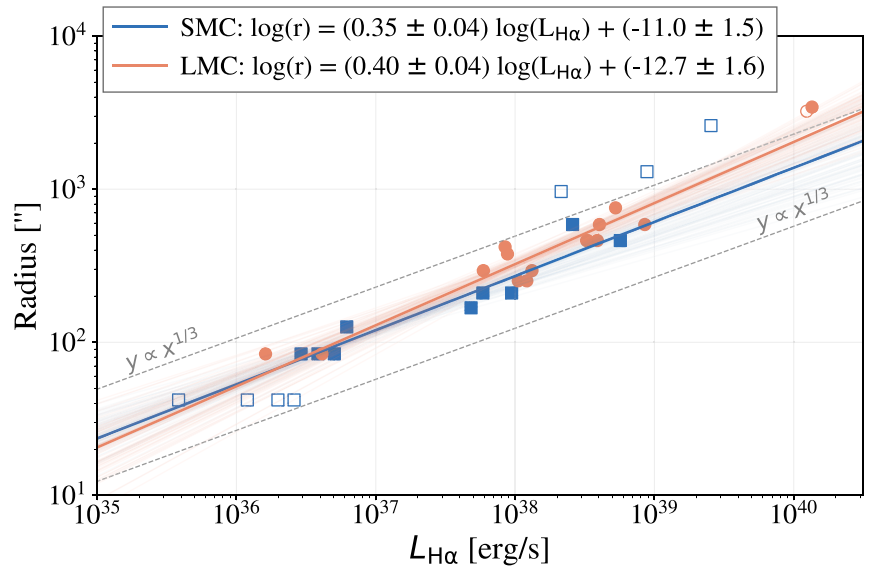

Figure 6. Correlations between the total $\mathrm{H} \alpha$ luminosity and the radius at which $\left\langle q_{\mathrm{PAH}}\right\rangle$ returns to the global average from the center of each $\mathrm{H}$ II region. The slope found in both cases is close to that expected for the expansion of a typical Strömgren sphere. We add a $y=x^{1 / 3}$ slope for comparison (gray dashed lines). (The empty symbols mark region where the calculated radius exceeds five times that given in Lopez et al. (2014), and are not used to fit the line. The light lines are results from bootstrapping for uncertainties on the coefficients.)

galaxies. If the $\mathrm{H} \alpha$ surface brightness were constant, growing the radius would lead to a dependence of $L_{\mathrm{H} \alpha}$ on $r^{2}$. We see in Figure 6 that is not the case. It rather suggests that the sphere of influence on the PAH fraction of the ionizing stars within an $\mathrm{H}$ II region scales as one would expect for the Strömgren sphere (Strömgren 1939). In this particular case, assuming a constant gas density the radius of the Strömgren sphere grows with the ionizing photon production rate (as does the $\mathrm{H} \alpha$ luminosity) with a power-law coefficient of one-third. If PAHs are destroyed in ionized gas by photodestruction or sputtering, then one would expect the region with a deficit of PAHs relative to the galaxy average to grow as the size of the Strömgren sphere. In Binder \& Povich (2018), the authors found a relation between the population of stars inside starforming regions of the MW, and the PAH fraction. They show that a single O6 star is less effective at destroying PAHs than a population of stars that extends to $\mathrm{O} 2 / \mathrm{O} 3$, and Wolf-Rayet stars. Glatzle et al. (2019) showed that the growth of H II regions is related to the dust content within, including $\mathrm{PAH}$ abundance, by impacting the ionization fronts. A more detailed study of $\mathrm{H}$ II regions would be interesting to possibly link the initial $\left\langle q_{\mathrm{PAH}}\right\rangle$ value at small radius in Figure 5, the properties of the ionizing star(s) within, and the expansion of $\mathrm{H}$ II regions.

\section{4. $q_{\mathrm{PAH}}$ and $\mathrm{H \alpha}$}

Even outside of the $\mathrm{H}$ II regions, we find a clear relation between increasing $\mathrm{H} \alpha$ emission and decreasing $\left\langle q_{\mathrm{PAH}}\right\rangle$. Figure 7 shows $\left\langle q_{\mathrm{PAH}}\right\rangle$ binned as a function of $\mathrm{H} \alpha$ surface brightness for the SMC (blue squares) and the LMC (orange circles). Both correlations tend to reach $\left\langle q_{\mathrm{PAH}}\right\rangle \sim 0 \%$ at high $I_{\mathrm{H} \alpha}$ values, independently of the average value of each galaxy. This agrees with the scenario where PAHs are destroyed in H II regions. In Figure 7 , we identify the value $I_{\mathrm{H} \alpha} \sim 1.5 \times 10^{-16} \mathrm{erg} \mathrm{s}^{-1} \mathrm{~cm}^{-2} \operatorname{arcsec}^{-2}$ (or $\sim 300 \mathrm{dR}$ ) as the $\mathrm{H} \alpha$ emission level at which $\left\langle q_{\mathrm{PAH}}\right\rangle$ starts to rapidly decrease in the LMC (dashed gray line). We use this value to plot the solid contours on Figure 4. This marks a limit where the PAH fraction drops more steeply with $\mathrm{H} \alpha$ surface brightness. We use this value as a separation between the diffuse neutral gas and ionized gas. Figure 7 shows that the PAH fraction decreases

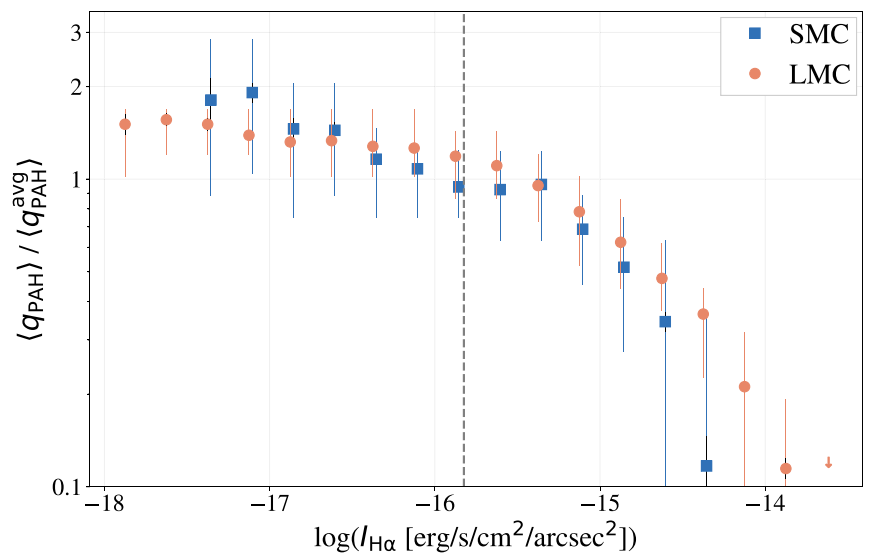

Figure 7. Correlation between $\left\langle q_{\mathrm{PAH}}\right\rangle$ and $\mathrm{H} \alpha$ surface brightness, for the LMC (orange circles) and the SMC (blue squares). The PAH fraction is normalized to the global average in each galaxy. Arrows indicate that the value falls below $\left\langle q_{\text {PAH }}\right\rangle=0.1$. The vertical dashed gray line marks the limit between the diffuse neutral and non-H II region ionized gas.

even at low values of $\mathrm{H} \alpha$ surface brightness, but that it drops rapidly only after it reaches the vertical dashed line $\left(\sim 1.5 \times 10^{-16} \mathrm{erg} \mathrm{s}^{-1} \mathrm{~cm}^{-2} \operatorname{arcsec}^{-2}\right.$, or $\left.300 \mathrm{dR}\right)$. Before that value, the PAH fraction decreases only mildly. To first order the $\mathrm{H} \alpha$ emission traces the surface density of ionized gas. Given that the distribution of $\mathrm{HI}$ is fairly flat across both galaxies (e.g., Stanimirovic et al. 2000), it is possible that the turn-over at $300 \mathrm{dR}$ occurs at a point where ionized gas starts to make a large contribution to the total surface density. The limit marked by the vertical dashed line is lower than the $\mathrm{H} \alpha$ surface brightness in $\mathrm{H}$ II regions, implying that PAHs may undergo destruction even in ionized gas outside the H II regions. ${ }^{15}$

A decrease of PAH fraction in DIG has been suggested by previous works on the MW by Dobler et al. (2009) and Dong \& Draine (2011). In Dong \& Draine, the authors studied the $\mathrm{H} \alpha$ to-free-free emission in the MW DIG, and found that this ratio corresponds to a lower temperature than they were able to produce with their model using the MW diffuse ISM PAH abundance. With a model consisting of ionized gas, recombining gas in the process of cooling, and cool neutral gas, they managed to reproduce the observations by lowering (by a factor of $\sim 3$ ) the PAH fraction in photoionized regions with respect to that of the global average in the ISM. Our results, constrained from observed SEDs, agree with a scenario where the PAH fraction is lower in ionized gas.

\section{5. $q_{\mathrm{PAH}}$ and the Radiation Field}

A high intensity of the radiation field is often quoted as a cause for enhanced destruction of PAHs. Here, we can test that scenario by looking at $\left\langle q_{\mathrm{PAH}}\right\rangle$ as a function of $U_{\mathrm{min}}$, or $\bar{U}$. In our work, we only adjust for the radiation field intensity and not the hardness. In the top panel of Figure 8, we show the variations of $\left\langle q_{\mathrm{PAH}}\right\rangle$ in bins of $\bar{U}$, in the diffuse neutral medium (filled symbols), and the non-H II region ionized gas (above the limit in $\mathrm{H} \alpha$ emission and outside of $\mathrm{H}$ II regions; empty symbols). We choose to compare the diffuse neutral medium and the non$\mathrm{H}$ II region ionized gas because ionized-gas-related destruction

\footnotetext{
15 Assuming an electron and proton density $n_{\mathrm{e}} \sim n_{\mathrm{p}}=0.5 \mathrm{~cm}^{-3}$, a gas temperature $T=5000 \mathrm{~K}$, and a flat $\mathrm{HI}$ surface density in the LMC $\Sigma_{\mathrm{H} \mathrm{I}}=20 \times 10^{20} \mathrm{~cm}^{-} 2$, our limit in $\mathrm{H} \alpha$ surface brightness corresponds to a fraction of ionized gas $\sim 12 \%$, or an ionized zone depth $\sim 150 \mathrm{pc}$.
} 


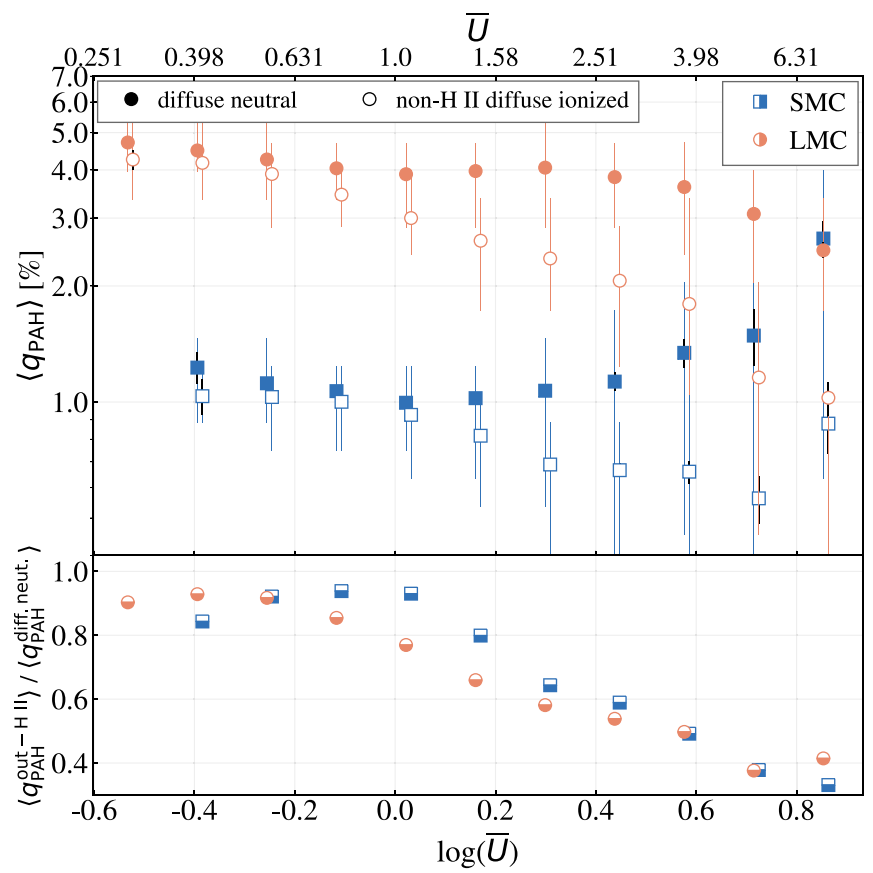

Figure 8. Top: variations of $\left\langle q_{\mathrm{PAH}}\right\rangle$ as a function of $\bar{U}$ derived from the fit, for the SMC (blue), and the LMC (orange). Pixels that fall above the limit in $\mathrm{H} \alpha$ emission, and outside of $\mathrm{H}$ II regions, are marked with empty symbols. Pixels that fall in the diffuse neutral medium, i.e., below $300 \mathrm{dR}$ and below the $\mathrm{CO}$ detection threshold, are marked with filled symbols. Bottom: fraction of $\left\langle q_{\mathrm{PAH}}\right\rangle$ in the ionized medium with respect to $\left\langle q_{\mathrm{PAH}}\right\rangle$ in the neutral medium.

processes should not occur in the former. In the diffuse neutral medium of the SMC, $\left\langle q_{\mathrm{PAH}}\right\rangle$ seems to increase slightly with $\bar{U}$ although with increasing uncertainty. This uptick in $\left\langle q_{\mathrm{PAH}}\right\rangle$ may be the result of an overestimation due to the fact that we do not take into account a change in radiation field hardness (see Section 5.3.1) as $\bar{U}$ increases. It is possible that, in lowmetallicity environments, changes in the radiation field hardness are more important, hence the observed increase of $\left\langle q_{\mathrm{PAH}}\right\rangle$ in the SMC. In the diffuse neutral medium in the LMC, however, we notice a decrease of $\left\langle q_{\mathrm{PAH}}\right\rangle$ at $\bar{U} \sim 3$. Given the scatter, there are only minor variations of $\left\langle q_{\mathrm{PAH}}\right\rangle$ up to $\log$ $(\bar{U})=0.5(\bar{U} \sim 3)$. At this point, $\left\langle q_{\text {PAH }}\right\rangle$ decreases noticeably. Below this, the intensity of the radiation field does not seem to affect the PAH fraction. As expected, in the ionized gas outside of $\mathrm{H}$ II regions, there seems to be a decrease of $\left\langle q_{\mathrm{PAH}}\right\rangle$ with $\bar{U}$ even for $\bar{U}<1$. This is not surprising because, in defining this phase, we selected the pixels where $\left\langle q_{\mathrm{PAH}}\right\rangle$ decreases in Figure 7 . When we control for the intensity of the radiation field, we can see that the gas phase has an impact on the PAH fraction. For an identical $\bar{U}$, the PAH fraction does not decrease as quickly, whether it is in the diffuse neutral or the non-H II region ionized gas. We also point out that, even at the lowest $\bar{U},\left\langle q_{\mathrm{PAH}}\right\rangle$ in the ionized medium never reaches that of the neutral medium.

The bottom panel of Figure 8 shows the ratio of $\mathrm{PAH}$ fraction in different gas phases $\left\langle q_{\mathrm{PAH}}^{\text {out }-\mathrm{H}}{ }_{\mathrm{II}}\right\rangle /\left\langle q_{\mathrm{PAH}}^{\text {diff. neut. }}\right\rangle$ (same color code as the top panel). It is interesting to notice the increasing offset between the PAH fraction in the ionized gas and the neutral medium as $\bar{U}$ increases, and that this offset is the same in both galaxies. We discuss these results in the context of PAH destruction in Section 5.2.

\section{Discussion}

\subsection{Dependence of $q_{\mathrm{PAH}}$ on Metallicity}

The difference between the global averages $\left\langle q_{\mathrm{PAH}}^{\mathrm{SMC}}\right\rangle$ and $\left\langle q_{\mathrm{PAH}}^{\mathrm{LMC}}\right\rangle$ is in good agreement with the power-law dependence of $q_{\text {PAH }}$ with metallicity found by Rémy-Ruyer et al. (2015). ${ }^{16}$ Our values also sit well within the scatter of the Draine et al. (2007) results, where a steep drop of $q_{\mathrm{PAH}}$ occurs at a metallicity threshold of $12+\log (\mathrm{O} / \mathrm{H}) \sim 8.1$.

The degree to which our results agree with either the threshold or power-law dependence, however, depends on the gas phase. In the MW, studies have found a diffuse neutral medium $q_{\text {PAH }}$ value of $\sim 4.6 \%$ (Li \& Draine 2001; Weingartner $\&$ Draine 2001a). For a fair comparison, this should be compared to our selection of the diffuse neutral medium in the LMC and SMC listed in Table 2. In this case, the power-law boundaries found by Rémy-Ruyer et al. (2015) underestimate the PAH fraction in the LMC. Indeed, the LMC's diffuse neutral gas $q_{\mathrm{PAH}}$ is very similar to the MW's, while the SMC falls a factor of $\sim 4$ lower. If we treat the diffuse neutral gas as a reference value, where most destruction processes are not operating, our results strengthen the interpretation that there is a threshold in metallicity where $q_{\mathrm{PAH}}$ decreases significantly, and that it lies between SMC and LMC metallicities.

\subsection{Insights into the Life-cycle of PAHs from Comparison of $\left\langle q_{\mathrm{PAH}}\right\rangle$ in Different Phases}

We use the separation of the gas phases in the MCs and their associated PAH fraction to investigate the life-cycle of PAHs in the ISM. We first go over some of the possible scenarios for $\mathrm{PAH}$ formation and how they might affect $\left\langle q_{\mathrm{PAH}}\right\rangle$ in each phase.

Theoretical studies have found that the atmospheres of postasymptotic giant branch (AGB) stars could be a favored site for the formation of PAHs (Cherchneff et al. 1992; Sloan et al. 2007). PAHs produced by AGB stars should primarily input into the diffuse ISM, since the old stellar distribution is not closely related to the current location of star-forming regions or dense gas (e.g., see Sandstrom et al. 2010 for a comparison between the SMC old stellar distribution and the ISM). If PAHs are primarily formed by AGB stars and no additional destruction or production mechanisms are operating in the diffuse neutral gas, one could argue that the $\left\langle q_{\mathrm{PAH}}^{\text {ref }}\right\rangle$ we have identified should reflect the efficiency of PAH formation by AGB stars. Boyer et al. $(2011,2012)$ studied the stellar population of both MCs, and found that the carbon-rich AGB population has similar dust production rates in the SMC and in the LMC, despite their different metallicities. Although other evolved stars such as red supergiants seem to be more effective at producing dust in the LMC, they do not play a major role in the overall dust production by evolved stars (e.g., Boyer et al. 2011). Therefore, without some additional destruction mechanism in the diffuse neutral phase, we cannot explain the different $\left\langle q_{\mathrm{PAH}}^{\text {ref }}\right\rangle$ between the SMC and the LMC solely with

\footnotetext{
${ }^{16}$ Their study deals with integrated SED fitting. Their $q_{\mathrm{PAH}}$ parameter is then similar to a luminosity-mass-weighted $\left\langle q_{\mathrm{PAH}}\right\rangle$ in our study.
} 
more effective formation of PAHs in AGB atmospheres in the LMC. ${ }^{17}$

Another scenario for PAH formation suggested by theoretical studies (e.g., Jones et al. 1996) is shattering of large carbon grains, leading to the production of smaller carbon grain fragments. Jones et al. (1996) argued that the shattering of carbon grains can occur at velocities as low as $1 \mathrm{~km} \mathrm{~s}^{-1}$, and is the prevalent process affecting dust grains at shock velocities $\geqslant 100 \mathrm{~km} \mathrm{~s}^{-1}$. Their study focused on the grain-grain interaction in the so-called warm intercloud medium, similar to our definition of diffuse neutral medium. There, they found that the redistribution of carbon-rich dust mass from large grains into small grains, and even PAH-like fragments, is significant. In Slavin et al. (2015), the authors found that the redistribution of dust mass in smaller grains is important in supernova remnants shocks up to $\sim 200 \mathrm{~km} \mathrm{~s}^{-1}$. A critical question, however, is whether the hot shocked gas is able to quickly sputter and destroy any PAH fragments that are created by shattering. Theoretical calculations by Micelotta et al. (2010b) suggest that PAHs are quickly destroyed by collisions with energetic particles at shock velocities higher than $100 \mathrm{~km} \mathrm{~s}^{-1}$. This work leads to the conclusion that shattering of dust grains, in $<100$ $\mathrm{km} \mathrm{s}^{-1}$ shocks, could be a significant source of PAH-like grains. Because the shattering process is directly linked to the available carbon-rich dust mass, one would expect a higher abundance of small grain fragments if there is a higher dust-togas ratio. Given the observed dust-to-gas ratio in the $\mathrm{MCs}$ $(\sim 0.003, \sim 0.0008$ in the LMC and SMC, respectively; Leroy et al. 2011; Gordon et al. 2014; Roman-Duval et al. 2014), this would be consistent with our finding of higher $\left\langle q_{\mathrm{PAH}}^{\text {ref }}\right\rangle$ in the $\mathrm{LMC}$, compared to that in the SMC. In the former, the higher $\left\langle q_{\mathrm{PAH}}^{\mathrm{ref}}\right\rangle$, assuming destruction of PAHs, is not significant in the diffuse neutral medium, and could be the result of more efficient shattering of large carbonaceous grains.

Another hypothesis for PAH formation is growth in the molecular phase of the ISM (e.g., Sandstrom et al. 2010, 2012; Zhukovska et al. 2016). In this scenario, the lower $\left\langle q_{\mathrm{PAH}}\right\rangle$ in the SMC would be related to less efficient growth processes in the molecular gas, possibly due to the lower abundance of metals to accrete onto existing grains to form PAHs. We do see that the $\left\langle q_{\mathrm{PAH}}^{\text {mol. }}\right\rangle$ is lower in the SMC than in the LMC. Previous work by Sandstrom et al. (2010) found enhanced $q_{\mathrm{PAH}}$ in dense regions of the SMC compared to the diffuse gas phases, which was interpreted as evidence for growth in the molecular gas and destruction operating in the diffuse ISM. We do find a higher $\mathrm{PAH}$ fraction in the molecular gas phase of each galaxy with respect to the global averages, (see Table 2; LMC: 4.3\%; SMC: $1.1 \%$ ), but the $\left\langle q_{\mathrm{PAH}}\right\rangle$ is similar in the molecular and diffuse neutral phases of each galaxy. The observation of the same $\left\langle q_{\mathrm{PAH}}\right\rangle$ in the molecular and diffuse neutral gas is consistent either with formation of PAHs in the diffuse ISM and incorporation into molecular clouds, or its inverse, assuming no destruction processes operate differentially in these two phases. In the same molecular gas, accretion and coagulation of small grains onto big grains (e.g., Stepnik et al. 2003; Köhler et al. 2012) could also lead to a decrease in the observed PAH fraction. In our case, observational evidence for that

\footnotetext{
17 If the PAH-like dust production is equal in both clouds, a higher production of non-PAH dust in the SMC would lead to a lower PAH fraction. Based on the same works previously quoted, there is no indication than the SMC stellar population is more effective at producing non-PAH dust than in the LMC, and we disfavor this possibility.
}

mechanism would be a lower PAH fraction in the molecular regions. Since we do not see such variation, we do not favor this possibility.

Observational studies have shown that the PAH size distribution and properties are sensitive to the local radiation field and gas ionization. This is seen in the vicinity of MW H II regions and photodissociation regions (e.g., Berné et al. 2007; Compiègne et al. 2007; Arab et al. 2012; Peeters et al. 2017) as well as in nearby galaxies (Gordon et al. 2008; Paradis et al. 2011; Relaño et al. 2016). PAH destruction can be accomplished in several ways: mediated by interaction with energetic photons, sputtering by particles in hot gas, or chemical reactions. A long-standing hypothesis to explain the PAH deficit at low metallicity is that PAHs are more readily destroyed in such conditions (e.g., Madden et al. 2006; Galliano et al. 2008). A possible scenario for enhanced destruction of PAHs at low metallicity is photodestruction by the radiation field, either due to increased intensity or hardness. There are a number of observational studies that have found correlations that agree with such a scenario. For example, Madden et al. (2006) and Gordon et al. (2008) found that the $\mathrm{PAH}$ features disappear as the radiation field hardness increases in $\mathrm{H}$ II regions (measured from MIR neon and sulfur line ratios). Theoretical studies have also shown that PAHs are subject to sputtering and fragmentation in ionized gas (Micelotta et al. 2010a; Bocchio et al. 2012) because of electronic and/or atomic interactions. There, projectile particles can reach a high velocity with respect to that of the grains, leading to catastrophic collisions. In general, the regions of the galaxy with the hardest radiation fields will also be those where ionized gas exists and the overall intensity of the radiation field is higher. Therefore, to distinguish between the potential destruction mechanisms, we need to attempt to separate the effects of these quantities. We do so using $\mathrm{H} \alpha$ emission as a tracer of ionized gas, and our determination of $\bar{U}$ as a tracer of the intensity of the radiation field, as presented in Section 4.5 and Figure 8. We do not have a direct tracer for the hardness of the radiation field covering the full extent of the galaxies. We note the similar trends of the ratios $\left\langle q_{\mathrm{PAH}}^{\text {out }-\mathrm{H}} \mathrm{II}\right\rangle /\left\langle q_{\mathrm{PAH}}^{\text {diff. neut. }}\right\rangle$ of the SMC (blue) and the LMC (orange), in the bottom panel of Figure 8. They suggest that metallicity does not have an impact on the relative efficiencies of the destruction processes between the diffuse neutral medium and the ionized gas outside of $\mathrm{H} \mathrm{II}$ regions (in the same bin of the radiation field). We do not test, however, the impact of metallicity on the global amount of each gas phase in a galaxy, which might have an impact on the overall destruction of PAHs.

PAHs in a more intense radiation field could be more easily destroyed because they are more fragile, due to their ionization state. Ionized PAHs are less stable, and more prone to losing, for example, $\mathrm{H}$ atoms (Montillaud et al. 2013). Weingartner \& Draine (2001b) showed that the charge of small carbonaceous grains varies depending on a parameter $G \sqrt{T} / n_{\mathrm{e}}$, where $G$ is the radiation field intensity, $T$ the temperature of the gas, and $n_{\mathrm{e}}$ the electron density. To test whether PAH ionization may lead to higher destruction rates, the different behavior of the neutral and ionized gas phases at fixed $\bar{U}$ from Figure 8 is of interest. In a bin of $\bar{U}$, we control for the radiation field intensity, and it is the same in both the diffuse neutral and the non-H II DIG. Weingartner \& Draine (2001b) showed that the gas temperature does not significantly affect the ionization state of PAHs. The only parameter remaining then is $n_{\mathrm{e}}$. If the electron density 
were lower in the non-H II ionized gas compared to the diffuse neutral gas, then PAHs could be more ionized in this medium, facilitating their destruction. While this seems counterintuitive, given that the non-H II region ionized gas is defined by its ionization state, the difference in overall density between the ionized and neutral phases and the low, but non-negligible, fractional ionization in the neutral medium could lead to the situation where $n_{\mathrm{e}}$ is lower for the ionized gas. Li \& Draine (2001) showed that PAHs larger than $7 \AA$ are more ionized in the MW's warm neutral medium than in the warm ionized medium. In that case, the lower $\left\langle q_{\mathrm{PAH}}^{\text {out }-\mathrm{H} \mathrm{II}}\right\rangle$ compared to that of the diffuse neutral medium could not be explained by an easier destruction of PAHs because of their ionization.

Based on the comparison between the gas phases in the two galaxies, we could explain the offset in $\left\langle q_{\mathrm{PAH}}\right\rangle$ between the LMC and SMC by: (i) fragmentation of large grains in the diffuse medium leading to more PAHs in the LMC due to its higher dust content; (ii) formation of PAHs in the molecular ISM and their injection in the diffuse gas, assuming there is no preferential destruction in either molecular or diffuse gas. The formation of PAHs in AGB stars cannot explain the difference in PAH fraction between the SMC and the LMC. The destruction mechanisms (e.g., photodestruction by the radiation field), suggested to be more effective in lower-metallicity galaxies, do not differ significantly between the SMC and the LMC in this work (see the bottom panel of Figure 8).

\subsection{Caveats}

\subsubsection{Impact of the Modeled Radiation Field}

The Draine \& Li (2007) model uses the radiation field described by Mathis et al. (1983), for the MW at the galactocentric distance $D_{\mathrm{G}}=10 \mathrm{kpc}$. This limits the possible variations allowed in our fitting. Specifically, the hardness of the radiation field is constant. However, it is expected that the relative proportion of UV versus optical photons will not be the same in H II regions where the ionizing stars produce more UV photons. Some studies have made adjustments to the radiation field to address this issue (e.g., Galliano et al. 2005; Salgado et al. 2016). In M33, Relaño et al. (2016) studied the dust content of $\mathrm{H}$ II regions, and specifically changed the radiation field to one with more UV photons. They showed that this leads to lower PAH fraction, by a factor up to 3. Paradis et al. (2011) studied the impact of the radiation field on dust emission fitting in the LMC by adding a $4 \mathrm{Myr}$ stellar population to the Mathis et al. (1983) radiation field. As expected, they found that, in $\mathrm{H}$ II regions, using a harder radiation field leads to a decrease in the PAH abundance estimation. This is linked to the PAH being more sensitive to the UV-visible part of the incident radiation field (Li \& Draine 2002). Harder radiation, i.e., more energetic photons, would enhance their MIR emission. In our study, this would make the offset between the SMC and the LMC (low-metallicity stars produce more UV photons; e.g., Eldridge et al. 2008), and the difference between diffuse-toionized PAH fraction, even more dramatic (this was also shown by Paradis et al. 2011, in the LMC).

\subsubsection{Diffuse Ionized Gas}

The definition of ionized gas in the MW (called "warm ionized gas"; e.g., Reynolds 1984; Madsen et al. 2006), and in extragalactic studies (called "DIG"; e.g., Zurita et al. 2000) is a delicate subject (see also McKee \& Begelman 1990;
Mathis 2000; Haffner et al. 2009). Given the difficulty defining "DIG," we decided not to make assumptions on the exact definition of the ionized medium outside of $\mathrm{H}$ II regions. A finer description of the local conditions of the ionized gas would require measurements of the electron/proton density, incident radiation field, and gas temperature.

\subsubsection{Metallicity Variations across the MCs}

In this study, we assume the metallicity to be constant across each galaxy. If there were metallicity variations, there should also be variations in $q_{\mathrm{PAH}}$. If the metallicity variations are not correlated with ISM phase, we would only expect to see enhanced scatter. If they were correlated with ISM phase, the results would likely be different. We do not see a good reason to assume any metallicity variations would be ISM phase correlated.

\section{Conclusions}

We fit the dust emission SED in the SMC and LMC using photometry from Spitzer and Herschel (3.6-500 $\mu \mathrm{m})$, with the dust emission model from Draine \& Li (2007). We provide maps of each fitting parameter at a $42^{\prime \prime}$ pixel size, i.e., $\sim 10 \mathrm{pc}$ in the LMC and $\sim 12 \mathrm{pc}$ in the SMC.

We especially focus on the spatial distribution of the PAH fraction $q_{\mathrm{PAH}}$, the fraction of dust mass in grains with less than $10^{3}$ carbon atoms. We find a global dust-mass-weighted PAH fraction $\left\langle q_{\mathrm{PAH}}^{\mathrm{SMC}}\right\rangle=1.0 \%$ and $\left\langle q_{\mathrm{PAH}}^{\mathrm{LMC}}\right\rangle=3.3 \%$, both lower than the MW diffuse neutral medium value $(4.6 \%)$.

We measure the PAH fraction in different gas phases, distinguished by ${ }^{12} \mathrm{CO}(J=1-0)$ and $\mathrm{H} \alpha$ emission (Table 2$)$ or the lack thereof. We use the diffuse neutral medium $\left(I_{\mathrm{H} \alpha} \lesssim 1.5 \times 10^{-16} \mathrm{erg} \mathrm{s}^{-1} \mathrm{~cm}^{-2} \operatorname{arcsec}^{-2}\right.$ and no molecular gas detection) as a reference value and discuss the relative $\left\langle q_{\mathrm{PAH}}\right\rangle$ in each gas phase. We find that the PAH fraction in the LMC diffuse neutral medium $(4.1 \%)$ is similar to that of the MW diffuse neutral ISM, while in the SMC it is substantially lower than in the MW (1.1\%). The galaxy-averaged $\left\langle q_{\mathrm{PAH}}\right\rangle$ are consistent with both a power-law dependence of $q_{\mathrm{PAH}}$ with metallicity (Rémy-Ruyer et al. 2015), as well as the existence of a threshold around $12+\log (\mathrm{O} / \mathrm{H}) \sim 8.1$ at which the PAH abundance changes rapidly (e.g., Draine et al. 2007). But $\left\langle q_{\mathrm{PAH}}\right\rangle$ in the diffuse neutral gas favors the latter hypothesis.

We find evidence that $\left\langle q_{\mathrm{PAH}}\right\rangle$ is systematically low in all identified H II regions (Figures 4, 7). Additionally, we show that the sphere of influence of the $\mathrm{H}$ II regions on the PAH fraction grows as one would expect for a Strömgren sphere (Figure 6).

We investigate possible metallicity-dependent $\mathrm{PAH}$ formation and destruction scenarios to explain the higher $\mathrm{PAH}$ fraction in the neutral medium of the LMC compared to the SMC.

1. We find higher $\left\langle q_{\mathrm{PAH}}\right\rangle$ in molecular gas with respect to the galaxy global averages, but similar to those of the diffuse neutral medium. This is consistent with formation of PAHs in the molecular gas and injection in the diffuse neutral medium, or vice versa.

2. In each galaxy, we find a clear trend of $q_{\mathrm{PAH}}$ with $\mathrm{H} \alpha$ emission on a global scale (Figure 7). We find a limit in $\mathrm{H} \alpha$ luminosity (or ionized gas fraction) at which the PAH abundance starts to decrease, and interpret this as a destruction of PAHs in the ionized gas. This limit is lower 
than the typical $\mathrm{H} \alpha$ emission in $\mathrm{H}$ II regions, suggesting that the ionized medium, even outside of $\mathrm{H}$ II regions, still affects the PAH fraction through destruction processes.

3. A radiation field intensity about twice that of the solar neighborhood seems to be enough to affect the PAH fraction, in both the neutral and ionized gas phase (Figure 8). However, the ionized medium always shows a lower PAH fraction than the neutral medium, even at equal intensity of the radiation field.

4. We find that formation of PAHs through the fragmentation of large grains is a plausible explanation for the higher $\left\langle q_{\mathrm{PAH}}\right\rangle$ in the LMC compared to the SMC diffuse neutral medium.

Future work will investigate the variations of the PAH fraction with metallicity in resolved, nearby galaxies. The launch of the James Webb Space Telescope will allow for the detection of PAHs at higher redshifts, and give us the opportunity to study the PAH fraction at low metallicity in the early universe.

We are very grateful to the referee for their careful reading and comments which greatly improved the paper. J.C. wishes to thank Jean-Philippe Bernard, Olivier Berné and Déborah Paradis for fruitful discussions. The work of J.C., K.S., I.C., A. K.L., and D.U. is supported by NASA ADAP grants NNX16AF48G and NNX17AF39G and National Science Foundation grant No. 1615728. The work of A.K.L. and D. U. is partially supported by the National Science Foundation under Grants No. 1615105, 1615109, and 1653300. This paper used the Southern H-Alpha Sky Survey Atlas (SHASSA), which is supported by the National Science Foundation.

Facility: Herschel, Spitzer.

\section{ORCID iDs}

Jérémy Chastenet (iD https://orcid.org/0000-0002-5235-5589 Karin Sandstrom (iD https://orcid.org/0000-0002-4378-8534 I-Da Chiang (江宜達) (10) https://orcid.org/0000-0003-2551-7148

Adam K. Leroy (iD https://orcid.org/0000-0002-2545-1700

Dyas Utomo (iD https://orcid.org/0000-0003-4161-2639

Caroline Bot (iD https://orcid.org/0000-0001-6118-2985

Karl D. Gordon (ib https://orcid.org/0000-0001-5340-6774

Bruce T. Draine (iD https://orcid.org/0000-0002-0846-936X

Kisetsu Tsuge (1D https://orcid.org/0000-0002-2794-4840

\section{References}

Allamandola, L. J., Tielens, A. G. G. M., \& Barker, J. R. 1985, ApJL, 290, L25 Allamandola, L. J., Tielens, A. G. G. M., \& Barker, J. R. 1989, ApJS, 71, 733 Aniano, G., Draine, B. T., Calzetti, D., et al. 2012, ApJ, 756, 138

Aniano, G., Draine, B. T., Gordon, K. D., \& Sandstrom, K. 2011, PASP, 123,1218

Arab, H., Abergel, A., Habart, E., et al. 2012, A\&A, 541, A19

Bakes, E. L. O., \& Tielens, A. G. G. M. 1994, ApJ, 427, 822

Bernard, J.-P., Reach, W. T., Paradis, D., et al. 2008, AJ, 136, 919

Berné, O., Joblin, C., Deville, Y., et al. 2007, A\&A, 469, 575

Binder, B. A., \& Povich, M. S. 2018, ApJ, 864, 136

Bocchio, M., Micelotta, E. R., Gautier, A.-L., \& Jones, A. P. 2012, A\&A, 545, A124

Bolatto, A. D., Simon, J. D., Stanimirović, S., et al. 2007, ApJ, 655, 212

Bot, C., Ysard, N., Paradis, D., et al. 2010, A\&A, 523, A20

Boyer, M. L., Srinivasan, S., Riebel, D., et al. 2012, ApJ, 748, 40

Boyer, M. L., Srinivasan, S., van Loon, J. T., et al. 2011, AJ, 142, 103

Bron, E., Le Bourlot, J., \& Le Petit, F. 2014, A\&A, 569, A100

Chastenet, J., Bot, C., Gordon, K. D., et al. 2017, A\&A, 601, A55
Cherchneff, I., Barker, J. R., \& Tielens, A. G. G. M. 1992, ApJ, 401, 269 Chiang, I.-D., Sandstrom, K. M., Chastenet, J., et al. 2018, ApJ, 865, 117 Compiègne, M., Abergel, A., Verstraete, L., et al. 2007, A\&A, 471, 205 Cortzen, I., Garrett, J., Magdis, G., et al. 2019, MNRAS, 482, 1618 Desert, F.-X., Boulanger, F., \& Puget, J. L. 1990, A\&A, 237, 215 Dobler, G., Draine, B., \& Finkbeiner, D. P. 2009, ApJ, 699, 1374 Dong, R., \& Draine, B. T. 2011, ApJ, 727, 35

Draine, B. T., Dale, D. A., Bendo, G., et al. 2007, ApJ, 663, 866

Draine, B. T., \& Li, A. 2007, ApJ, 657, 810

Eldridge, J. J., Izzard, R. G., \& Tout, C. A. 2008, MNRAS, 384, 1109 Engelbracht, C. W., Gordon, K. D., Rieke, G. H., et al. 2005, ApJL, 628, L29

Fazio, G. G., Hora, J. L., Allen, L. E., et al. 2004, ApJS, 154, 10

Fukui, Y., Kawamura, A., Minamidani, T., et al. 2008, ApJS, 178, 56

Fukui, Y., Ogawa, H., Kawabata, K., Mizuno, A., \& Sugitani, K. 1991, in IAU Symp. 148, The Magellanic Clouds, ed. R. Haynes \& D. Milne (Cambridge: Cambridge Univ. Press), 105

Fukui, Y., Tsuge, K., Sano, H., et al. 2017, PASJ, 69, L5

Galliano, F., Dwek, E., \& Chanial, P. 2008, ApJ, 672, 214

Galliano, F., Hony, S., Bernard, J.-P., et al. 2011, A\&A, 536, A88

Galliano, F., Madden, S. C., Jones, A. P., Wilson, C. D., \& Bernard, J.-P. 2005, A\&A, 434, 867

Gaustad, J. E., McCullough, P. R., Rosing, W., \& Van Buren, D. 2001, PASP, 113,1326

Glatzle, M., Ciardi, B., \& Graziani, L. 2019, MNRAS, 482, 321

Gordon, K. D., Clayton, G. C., Misselt, K. A., Landolt, A. U., \& Wolff, M. J. 2003, ApJ, 594, 279

Gordon, K. D., Engelbracht, C. W., Rieke, G. H., et al. 2008, ApJ, 682, 336

Gordon, K. D., Meixner, M., Meade, M. R., et al. 2011, AJ, 142, 102

Gordon, K. D., Roman-Duval, J., Bot, C., et al. 2014, ApJ, 797, 85

Graczyk, D., Pietrzyński, G., Thompson, I. B., et al. 2014, ApJ, 780, 59

Griffin, M. J., Abergel, A., Abreu, A., et al. 2010, A\&A, 518, L3

Haffner, L. M., Dettmar, R.-J., Beckman, J. E., et al. 2009, RvMP, 81, 969

Israel, F. P., Wall, W. F., Raban, D., et al. 2010, A\&A, 519, A67

Jameson, K. E., Bolatto, A. D., Leroy, A. K., et al. 2016, ApJ, 825, 12

Jones, A. P., Tielens, A. G. G. M., \& Hollenbach, D. J. 1996, ApJ, 469, 740

Jones, O. C., Woods, P. M., Kemper, F., et al. 2017, MNRAS, 470, 3250

Köhler, M., Stepnik, B., Jones, A. P., et al. 2012, A\&A, 548, A61

Le Bourlot, J., Le Petit, F., Pinto, C., Roueff, E., \& Roy, F. 2012, A\&A, 541, A76

Le Page, V., Snow, T. P., \& Bierbaum, V. M. 2009, ApJ, 704, 274

Leger, A., \& Puget, J. L. 1984, A\&A, 137, L5

Leroy, A., Bolatto, A., Stanimirovic, S., et al. 2007, ApJ, 658, 1027

Leroy, A. K., Bolatto, A., Gordon, K., et al. 2011, ApJ, 737, 12

Li, A., \& Draine, B. T. 2001, ApJ, 554, 778

Li, A., \& Draine, B. T. 2002, ApJ, 572, 232

Lopez, L. A., Krumholz, M. R., Bolatto, A. D., et al. 2014, ApJ, 795, 121

Madden, S. C., Galliano, F., Jones, A. P., \& Sauvage, M. 2006, A\&A, 446, 877

Madsen, G. J., Reynolds, R. J., \& Haffner, L. M. 2006, ApJ, 652, 401

Mathis, J. S. 1994, ApJ, 422, 176

Mathis, J. S. 2000, ApJ, 544, 347

Mathis, J. S., Mezger, P. G., \& Panagia, N. 1983, A\&A, 128, 212

McKee, C. F., \& Begelman, M. C. 1990, ApJ, 358, 392

Meixner, M., Gordon, K. D., Indebetouw, R., et al. 2006, AJ, 132, 2268

Meixner, M., Panuzzo, P., Roman-Duval, J., et al. 2013, AJ, 146, 62

Meixner, M., Panuzzo, P., Roman-Duval, J., et al. 2015, AJ, 149, 88

Micelotta, E. R., Jones, A. P., \& Tielens, A. G. G. M. 2010a, A\&A, 510, A37

Micelotta, E. R., Jones, A. P., \& Tielens, A. G. G. M. 2010b, A\&A, 510, A36

Mizuno, N., Rubio, M., Mizuno, A., et al. 2001, PASJ, 53, L45

Montillaud, J., Joblin, C., \& Toublanc, D. 2013, A\&A, 552, A15

Paradis, D., Paladini, R., Noriega-Crespo, A., et al. 2011, ApJ, 735, 6

Paradis, D., Reach, W. T., Bernard, J.-P., et al. 2009, AJ, 138, 196

Peeters, E., Bauschlicher, C. W., Jr., Allamandola, L. J., et al. 2017, ApJ, 836, 198

Peeters, E., Spoon, H. W. W., \& Tielens, A. G. G. M. 2004, ApJ, 613, 986

Pilbratt, G. L., Riedinger, J. R., Passvogel, T., et al. 2010, A\&A, 518, L1

Poglitsch, A., Waelkens, C., Geis, N., et al. 2010, A\&A, 518, L2

Relaño, M., Kennicutt, R., Lisenfeld, U., et al. 2016, A\&A, 595, A43

Rémy-Ruyer, A., Madden, S. C., Galliano, F., et al. 2015, A\&A, 582, A121

Reynolds, R. J. 1984, ApJ, 282, 191

Rieke, G. H., Young, E. T., Engelbracht, C. W., et al. 2004, ApJS, 154, 25

Roman-Duval, J., Gordon, K. D., Meixner, M., et al. 2014, ApJ, 797, 86

Russell, S. C., \& Dopita, M. A. 1992, ApJ, 384, 508

Sajina, A., Spoon, H., Yan, L., et al. 2009, ApJ, 703, 270

Salgado, F., Berné, O., Adams, J. D., et al. 2016, ApJ, 830, 118

Sandstrom, K. M., Bolatto, A. D., Bot, C., et al. 2012, ApJ, 744, 20 
Sandstrom, K. M., Bolatto, A. D., Draine, B. T., Bot, C., \& Stanimirović, S. 2010, ApJ, 715, 701

Shipley, H. V., Papovich, C., Rieke, G. H., Brown, M. J. I., \& Moustakas, J. 2016, ApJ, 818, 60

Siana, B., Smail, I., Swinbank, A. M., et al. 2009, ApJ, 698, 1273

Slavin, J. D., Dwek, E., \& Jones, A. P. 2015, ApJ, 803, 7

Sloan, G. C., Jura, M., Duley, W. W., et al. 2007, ApJ, 664, 1144

Stanimirovic, S., Staveley-Smith, L., van der Hulst, J. M., et al. 2000, MNRAS, 315,791

Steglich, M., Jäger, C., Rouillé, G., et al. 2010, ApJL, 712, L16

Stepnik, B., Abergel, A., Bernard, J.-P., et al. 2003, A\&A, 398, 551

Strömgren, B. 1939, ApJ, 89, 526

Tchernyshyov, K., Meixner, M., Seale, J., et al. 2015, ApJ, 811, 78

Tielens, A. G. G. M. 2008, ARA\&A, 46, 289

Tsuge, K., Sano, H., Tachihara, K., et al. 2019, ApJ, 871, 44
Utomo, D., Chiang, I., Leroy, A. K., Sandstrom, K. M., \& Chastenet, J. 2019, ApJ, 874, 141

Walker, A. R. 2012, Ap\&SS, 341, 43

Weingartner, J. C., \& Draine, B. T. 2001a, ApJ, 548, 296

Weingartner, J. C., \& Draine, B. T. 2001b, ApJS, 134, 263

Werner, M. W., Roellig, T. L., Low, F. J., et al. 2004, ApJS, 154,

Wolfire, M. G., Hollenbach, D., McKee, C. F., Tielens, A. G. G. M., \& Bakes, E. L. O. 1995, ApJ, 443, 152

Woods, P. M., Oliveira, J. M., Kemper, F., et al. 2011, MNRAS, 411, 1597

Zaritsky, D., Harris, J., Thompson, I. B., \& Grebel, E. K. 2004, AJ, 128, 1606 Zaritsky, D., Harris, J., Thompson, I. B., Grebel, E. K., \& Massey, P. 2002, AJ, 123,855

Zhukovska, S., Dobbs, C., Jenkins, E. B., \& Klessen, R. S. 2016, ApJ, 831, 147

Zurita, A., Rozas, M., \& Beckman, J. E. 2000, A\&A, 363, 9 